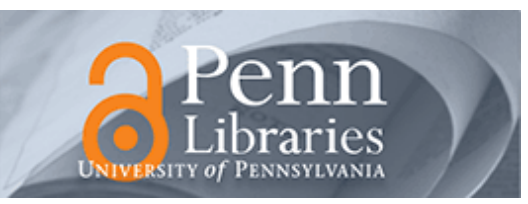

University of Pennsylvania

ScholarlyCommons

September 2002

\title{
Channel Assignment Algorithms Satisfying Cochannel and Adjacent Channel Reuse Constraints in Cellular Mobile Networks
}

\author{
Saswati Sarkar \\ University of Pennsylvania, swati@seas.upenn.edu \\ Kumar N. Sivarajan \\ Tejas Networks
}

Follow this and additional works at: https://repository.upenn.edu/ese_papers

\section{Recommended Citation}

Saswati Sarkar and Kumar N. Sivarajan, "Channel Assignment Algorithms Satisfying Cochannel and Adjacent Channel Reuse Constraints in Cellular Mobile Networks", . September 2002.

Copyright 2002 IEEE. Reprinted from IEEE Transactions on Vehicular Technology, Volume 51, Issue 5, September 2002, pages 954-967.

Publisher URL: http://ieeexplore.ieee.org/xpl/tocresult.jsp?i sNumber=24316\&puNumber=25

This material is posted here with permission of the IEEE. Such permission of the IEEE does not in any way imply IEEE endorsement of any of the University of Pennsylvania's products or services. Internal or personal use of this material is permitted. However, permission to reprint/republish this material for advertising or promotional purposes or for creating new collective works for resale or redistribution must be obtained from the IEEE by writing to pubs-permissions@ieee.org. By choosing to view this document, you agree to all provisions of the copyright laws protecting it.

This paper is posted at ScholarlyCommons. https://repository.upenn.edu/ese_papers/36

For more information, please contact repository@pobox.upenn.edu. 


\title{
Channel Assignment Algorithms Satisfying Cochannel and Adjacent Channel Reuse Constraints in Cellular Mobile Networks
}

\author{
Abstract \\ Improved channel assignment algorithms for cellular networks were designed by modeling the \\ interference constraints in terms of a hypergraph [1]. However, these algorithms only considered \\ cochannel reuse constraints. Receiver filter responses impose restrictions on simultaneous adjacent \\ channel usage in the same cell or in neighboring cells. We first present some heuristics for designing \\ fixed channel assignment algorithms with a minimum number of channels satisfying both cochannel and \\ adjacent channel reuse constraints. An asymptotically tight upper bound for the traffic carried by the \\ system in the presence of arbitrary cochannel and adjacent channel use constraints was developed in [2]. \\ However, this bound is computationally intractable even for small systems like a regular hexagonal \\ cellular system of 19 cells. We have obtained approximations to this bound using the optimal solutions \\ for cochannel reuse constraints only and a further graph theoretic approach. Our approximations are \\ computationally much more efficient and have turned out to track very closely the exact performance \\ bounds in most cases of interest.

\section{Keywords} \\ Adjacent channel interference, cellular systems, channel assignment algorithms, cochannel interference, \\ graph models, hypergraph models

\section{Comments} \\ Copyright 2002 IEEE. Reprinted from IEEE Transactions on Vehicular Technology, Volume 51, Issue 5, \\ September 2002, pages 954-967. \\ Publisher URL: http://ieeexplore.ieee.org/xpl/tocresult.jsp?i sNumber=24316\&puNumber=25 \\ This material is posted here with permission of the IEEE. Such permission of the IEEE does not in any way \\ imply IEEE endorsement of any of the University of Pennsylvania's products or services. Internal or \\ personal use of this material is permitted. However, permission to reprint/republish this material for \\ advertising or promotional purposes or for creating new collective works for resale or redistribution must \\ be obtained from the IEEE by writing to pubs-permissions@ieee.org. By choosing to view this document, \\ you agree to all provisions of the copyright laws protecting it.
}




\title{
Channel Assignment Algorithms Satisfying Cochannel and Adjacent Channel Reuse Constraints in Cellular Mobile Networks
}

\author{
Saswati Sarkar and Kumar N. Sivarajan, Member, IEEE
}

\begin{abstract}
Improved channel assignment algorithms for cellular networks were designed by modeling the interference constraints in terms of a hypergraph [1]. However, these algorithms only considered cochannel reuse constraints. Receiver filter responses impose restrictions on simultaneous adjacent channel usage in the same cell or in neighboring cells. We first present some heuristics for designing fixed channel assignment algorithms with a minimum number of channels satisfying both cochannel and adjacent channel reuse constraints. An asymptotically tight upper bound for the traffic carried by the system in the presence of arbitrary cochannel and adjacent channel use constraints was developed in [2]. However, this bound is computationally intractable even for small systems like a regular hexagonal cellular system of 19 cells. We have obtained approximations to this bound using the optimal solutions for cochannel reuse constraints only and a further graph theoretic approach. Our approximations are computationally much more efficient and have turned out to track very closely the exact performance bounds in most cases of interest.
\end{abstract}

Index Terms-Adjacent channel interference, cellular systems, channel assignment algorithms, cochannel interference, graph models, hypergraph models.

\section{INTRODUCTION}

$\mathbf{I}$ $\mathrm{N}$ A cellular system, the coverage area is logically divided into cells. Each cell has a cell site or a base station. The communication from the mobile user is directed to a central switching office by the base station. The central switching office directs this communication to the destination. Depending on the mode of multiple access used by the mobile customers, cellular systems can be broadly classified into channelized and nonchannelized systems. In a channelized cellular system the multiple access is time division multiple access (TDMA) or frequency division multiple access (FDMA) or a combination of both. The term channel refers to a time slot in TDMA, a frequency slot in FDMA, and a combination of both in TDMA/FDMA systems like group special mobiles (GSM). The traffic in a cellular systems is usually too high to allow the use of a channel for one

Manuscript received February 21, 1999; revised August 22, 2001. The work of S. Sarkar was supported in part by NSF Grant ANI01-06984. A part of this work was conducted at the Indian Institute of Science, Bangalore, supported by a Nortel Networks Grant, presented at the 34th Annual Allerton Conference on Communications, Control, and Computing 1996, and published in the Proceedings.

S. Sarkar is with the Department of Electrical Engineering, University of Pennsylvania, Philadelphia, PA 19104 USA (e-mail: swati@ee.upenn.edu).

K. N. Sivarajan is with Tejas Networks, Bangalore 560 0012, India (e-mail: kumar@tejasnetworks.com).

Digital Object Identifier 10.1109/TVT.2002.801768 call at a time; radio channels must be used simultaneously for more than one call. This is known as channel reuse. Channel reuse causes interference, which in turn degrades the transmission quality. Transmission quality requirements impose certain cochannel reuse constraints, i.e., the same channels may not be used simultaneously within certain distance.

Interference is also caused by simultaneous use of adjacent channels in close proximity. This happens because of imperfections in filters. Use of guard band between adjacent frequencies somewhat mitigates this interference. However, guard bands need to be significantly large in order to reduce this interference below an acceptable threshold. This leads to poor utilization of the limited radio spectrum. A better strategy is to eliminate the adjacent channel interference by not using adjacent channels in neighboring cells at the same time. This results in better use of radio spectrum as all available channels can be used in accommodating calls in the system. However, in this case, channel allocation strategies need to satisfy cochannel and adjacent channel use constraints. While frequency allocation in presence of cochannel reuse constraint has received significant attention, efficient channel allocation strategies which satisfy both cochannel and adjacent channel use constraints do not exist. This paper is directed toward addressing both these constraints.

We first describe our system model and the existing theoretical results for cellular systems with different types of channel allocation constraints, e.g., cochannel reuse constraints only [4], and both cochannel and adjacent channel use constraints [2] (Section II). Next, we present efficient fixed channel allocation strategies that attain low blocking probabilities in presence of limited spectrum availability in systems with cochannel and adjacent channel constraints (Section III). The channel allocation strategy we present closely approximates the minimum number of channels required to attain certain desired blocking probabilities in cells. Performance bounds are known for cellular systems with different channel use constraints. The efficiency of actual channel allocation strategies can be determined by comparing their performances with these bounds. However, these bounds are computationally complex when both cochannel and adjacent channel constraints are considered. We present computationally simple approximation for these bounds, which track the exact bounds closely (Sections IV and V). Channel use constraints need not be limited to adjacent channels, but may extend to simultaneous use of arbitrary channels. This is because transmissions in nonadjacent channels may also cause interference. However, the interference produced by simultaneous use of 
TABLE I

SYMBOL TABLE

\begin{tabular}{|l|l|}
\hline Symbol & Meaning \\
\hline $\mathrm{N}$ & Number of cells \\
\hline $\mathrm{M}$ & $\begin{array}{l}\text { Number of maximal independent sets } \\
\text { of the hypergraph modeling co-channel } \\
\text { reuse constraints }\end{array}$ \\
\hline$a_{i j}$ & $\begin{array}{l}\text { Indicates whether cell } i \text { is in } j \text { th maximal } \\
\text { independent set }\end{array}$ \\
\hline$b_{i j}$ & $\begin{array}{l}\text { Indicates whether cells } i \text { and } j \text { can use an } \\
\text { adjacent channel simultaneously }\end{array}$ \\
\hline$r$ & Offered traffic \\
\hline$p_{i}$ & Fraction of offered traffic for cell $i$ \\
\hline$\tau$ & Number of possible states of a channel \\
\hline$R$ & Number of hyperstates of a channel \\
\hline$r_{0}$ & $\begin{array}{l}\text { System capacity without adjacent channel } \\
\text { use constraints }\end{array}$ \\
\hline$T(r)$ & $\begin{array}{l}\text { Traffic carried by system in absence of } \\
\text { adjacent channel use constraints }\end{array}$ \\
\hline$r_{0}^{A}$ & $\begin{array}{l}\text { System capacity with adjacent channel } \\
\text { use constraints }\end{array}$ \\
\hline$T_{A}(r)$ & $\begin{array}{l}\text { Traffic carried by system in presence of } \\
\text { adjacent channel use constraints }\end{array}$ \\
\hline$r_{0}^{A L}$ & Lower bound to $r_{0}^{A}$ \\
\hline$T_{A L}(r)$ & Lower bound to $T_{A}(r)$ \\
\hline
\end{tabular}

nonadjacent channels in vicinity is negligible in general. Nevertheless, this interference may be significant in some cases, and as such we address this generalization in Section VI.

\section{System Model And AnAlytical Performance Bounds}

We describe the system model in this section. We introduce some notations for this purpose. Table I summarizes all notations used extensively in the paper. The system consists of $N$ cells which share a common set of $n$ channels. The underlying offered traffic model is independent from cell to cell; in particular, we ignore the effects of call handovers and inter-cell calls. However, it is likely that we can extend our results to the case in which this independence assumption is dropped and handovers and intercell calls are included. Our optimism is derived from the fact that the results of [4], to which we shall refer extensively, have been extended to include handovers in [7]. The underlying model of offered traffic satisfies the "asymptotic traffic property" (ATP) [4], which states that

$$
\lim _{n \rightarrow \infty} C(k, n) / n=\min (r, 1), \quad \text { where } \lim _{n \rightarrow \infty} k / n=r .
$$

$C(k, n)$ is the carried traffic in a system with one cell, when the offered traffic is $k$ and the number of available channels is $n$. Many offered traffic models including the common Poisson arrivals and exponential holding times satisfy the ATP.

The expected number of calls that would be in progress in cell $i$ if all call requests could be honored is known as the offered traffic in cell $i$. If $A_{i}$ denotes the offered traffic in cell $i$ then $A_{i} / n$ is the offered traffic intensity in cell $i$. The offered traffic intensity in the system, $r$, is the sum of the offered traffic intensities in the cells; thus, $r=\sum A_{i} / n$. We assume that $r$ is rational. The ratio $p_{i}=A_{i} / \sum_{i=1}^{N} A_{i}$ represents the fraction of the total offered traffic in cell $i$ and the vector $\tilde{p}=\left(p_{1}, p_{2}, \ldots, p_{N}\right)$ is the traffic pattern. We assume that the $p_{i} \mathrm{~s}$ are rational. The carried traffic intensity in cell $i, x_{i}$, is the carried traffic (expected number of calls in progress) in cell $i$ per available channel in the system. An objective is to maximize the total traffic carried by a system $\sum_{i=1}^{N} x_{i}$.

We will first describe a mathematical model and performance bounds for systems with cochannel reuse constraints only. These have been presented in [4]. Subsequently, we present a generalization for including the adjacent channel use constraints. This generalization was developed in [2].

Mathematical Model for Systems With Cochannel Reuse Constraints: Cochannel reuse constraints in cellular systems have been modeled by regular hexagonal channel reuse patterns [3] for a long time. A hypergraph model was first used in [4]. This has been found to be the most efficient model for cochannel reuse constraints [5]. We first describe the hypergraph model briefly. A hypergraph $H$ is formally defined as $H=(V, E)$, where $V$ is the set of vertices and $E$ is the set of edges, where each edge $e$ is a nonempty subset of $V$ such that $\bigcup_{e \in E} e=V$ [6]. A hypergraph is a generalization of a graph in that an edge can have no more than two vertices in a graph but this restriction does not hold for a hypergraph. Hypergraph modeling of cellular systems is as follows:

- Each cell corresponds to a vertex.

- A forbidden set is a group of cells all of which cannot use a channel simultaneously. If no proper subset of a forbidden set is forbidden, then it is a minimal forbidden set. An edge is a minimal forbidden set.

- A set of vertices which does not contain an edge is an independent set. Any group of cells which may use the same channel simultaneously forms an independent set of the underlying hypergraph. If an independent set is not a proper subset of another independent set, then it is a maximal independent set.

Let the hypergraph $H$ modeling the cochannel reuse constraints have $M$ maximal independent sets and let $N_{j}$ denote the size of the $j$ th maximal independent set. We define

$$
a_{i j}= \begin{cases}1, & \begin{array}{l}
\text { if the } i \text { th cell is in the } j \text { th maximal } \\
\text { independent set of the hypergraph modeling } \\
\text { the cochannel reuse constraints and }
\end{array} \\
0, & \text { otherwise. }\end{cases}
$$

Now, [4] presents an asymptotically ${ }^{1}$ tight upper bound on the total carried traffic intensity in the cellular system with cochannel reuse constraints. The upper bound $T(r)$ is a function of the offered traffic $r$ and is given by the optimal value of the objective function of the following linear program:

Maximize

$$
\sum_{i=1}^{N} x_{i}
$$

subject to

$$
\left.\begin{array}{ll}
x_{i} \leq \sum_{j=1}^{M} X_{j} a_{i j}, & i=1,2, \ldots, N \\
x_{i} \leq p_{i} r, & i=1,2, \ldots, N \\
X_{j} \geq 0, & j=1,2, \ldots, M \\
\sum_{j=1}^{M} X_{j}=1 &
\end{array}\right\}
$$

${ }^{1}$ The number of channels and the offered traffic are made arbitrarily large while keeping the ratio finite. 
We denote this linear program by LP1. The intuition behind LP1 can be described as follows. The variable $x_{i}$ denotes the traffic carried in cell $i$. The objective is to maximize the sum of the traffic carried in all cells. Note that a channel can be simultaneously used by all cells in an independent set. Thus, one can think of assigning channels to maximal independent sets rather than to cells. Whenever a channel is assigned to a maximal independent set, all cells in the set receives the channel. Here, $X_{j}$ denotes the fraction of channels allocated to the $j$ th maximal independent set. Thus, $\sum_{j=1}^{M} X_{j} a_{i j}$ denotes the fraction of channels allotted to the maximal independent sets which contain cell $i$ and this is the channel allocation of cell $i$. Clearly, the carried traffic in cell $i, x_{i}$ is upper bounded by this quantity. The first constraint represents this condition. The second constraint states that the carried traffic in a cell is upper bounded by the offered traffic. The remaining constraints are intuitive. This linear program has $M+N$ nonnegative variables and $2 N+1$ constraints.

It was proved in [4] that if the offered traffic intensity, $r$, is less than or equal to a certain quantity $r_{0}$, which depends on the cellular system and the traffic pattern and there is no adjacent channel use constraint, then there exists a channel assignment algorithm which achieves arbitrarily low blocking probabilities, if the number of available channels is sufficiently large. For $r>r_{0}$, no channel assignment algorithm can produce zero blocking for any number of channels. The quantity $r_{0}$ has been termed the capacity of the system. The capacity of a system is a measure of the reuse offered by the system as informally speaking each channel can carry $r_{0}$ calls simultaneously in the system on an average. It can also serve as a good operating load. The capacity, $r_{0}$ is given by the optimal value of the objective function of the following linear program:

$$
\max \left\{\begin{array}{c}
r: \sum_{j=1}^{M} X_{j}=1, X_{j} \geq 0, \sum_{j=1}^{M} X_{j} a_{i j} \geq p_{i} r, \\
i=1, \ldots, N
\end{array}\right\} \text { (LP2). }
$$

We denote this linear program by LP2. Intuitively, $r_{0}$ is the largest value of offered traffic $r$ for which the offered traffic in any cell $i, p_{i} r$ does not exceed the resource allocated to cell $i, \sum_{j=1}^{M} X_{j} a_{i j}$. This linear program has $M+1$ nonnegative variables and $N+1$ constraints.

The carried traffic $T(r)$ and the system capacity $r_{0}$ are important performance metrics of a cellular system. Now, [2] computes these metrics in presence of adjacent channel use constraints. However, the strategies presented there are computationally complex. An important contribution of this paper is to approximate these computations using techniques that are computationally simple. For this purpose, we will use the optimum solutions of LP1 and LP2 and a further graph theoretic technique. First, we explain the exact computations presented in [2].

Mathematical Model for Systems With Cochannel and Adjacent Channel Use Constraints: Imperfect receiver filter responses impose restrictions on simultaneous use of adjacent channels in nearby cells. By "adjacent" channels we mean consecutive carrier frequencies in an FDMA system. In the case of FDMA/TDMA systems, we assume all the time slots in a fre- quency channel are allocated to the same cell so that we can treat each carrier frequency as a channel. In the rest of this paper, we assume that a channel is a carrier frequency. The $n$ channels are numbered $1,2, \ldots, n$ with adjacent channels given consecutive numbers. The adjacent channel use constraints can be modeled by an $N \times N$ matrix $B$ such that

$$
b_{i j}= \begin{cases}1, & \text { if the } i \text { th and the } j \text { th cells can use } \\ \text { adjacent channels simultaneously and } \\ 2, & \text { otherwise. }\end{cases}
$$

The matrix $B$ can be determined from the transmission quality requirements and the interference produced by adjacent channels. For example, if the filters are perfect, then the adjacent channels do not interfere with each other. As such, any two cells can use adjacent channels simultaneously. In this case, $b_{i j}=1$ for all $i, j$. Typically, the cells within a certain distance $d$ cannot use adjacent channels simultaneously. Thus, $b_{i j}=1$ if cells $i$ and $j$ are separated by a distance greater than some $d$ and $b_{i j}=2$ otherwise. The value of $d$ depends on the interference caused by adjacent channels and the transmission quality requirements. Higher the interference or lesser the acceptable interference threshold, higher the value of $d$. Typical values of $d$ are zero and $\sqrt{3} P$ in regular hexagonal cellular systems, where $P$ is the cell radius. If $d=0$, adjacent channels cannot be used simultaneously in the same cell and if $d=\sqrt{3} P$ adjacent channels cannot be used simultaneously in the same cell and in adjacent cells.

Now [2] presents asymptotically tight upper bounds on the carried traffic intensity and the system capacity in presence of cochannel and adjacent channel use constraints. We introduce some notations for describing the results. The state of a channel is an $N$-tuple whose elements are either zero or one. The $i$ th element is one iff the channel is carrying a call in the $i$ th cell. The state of a channel (other than the one represented by the all zero $N$-tuple) represents an independent set of the underlying hypergraph modeling the cochannel reuse constraints. Let $\tau$ denote the set of states of a channel. (If the cochannel reuse constraints are modeled by a hypergraph, the elements of $\tau$, with the exception of the all zero $N$-tuple, have a one-to-one correspondence with the set of independent sets of the hypergraph.) The hyperstate of a channel $i, 1 \leq i<n$, is $(p, q)$, if the channel $i$ is in state $p$ and the channel $i+1$ is in state $q . \Omega \subseteq \tau \times \tau$ is the set of hyperstates of a channel. ${ }^{2} d_{i \omega}=1$ iff a channel carries a call in cell $i$ in the hyperstate $\omega \in \Omega$. $t_{\omega}=\sum_{i=1}^{N} d_{i \omega}$. Let $|\Omega|=R$. $\Omega_{U}(\eta)=\left\{\omega: \omega=\left(\eta, \eta^{\prime}\right) \in \Omega, \eta^{\prime} \in \tau\right\}$, and $\Omega_{L}(\eta)=$ $\left\{\omega: \omega=\left(\eta^{\prime}, \eta\right) \in \Omega, \eta^{\prime} \in \tau\right\}, \eta \in \tau . \Omega_{U}(\eta), \Omega_{L}(\eta) \subseteq \Omega$. An asymptotically tight upper bound $T_{A}(r)^{3}$ on the total carried traffic intensity in the system in the presence of cochannel and adjacent channel use constraints can be computed as follows:

$$
T_{A}(r)=\max \left\{\sum_{\omega \in \Omega} t_{\omega} s_{\omega}: s=\left(s_{1}, s_{2}, \ldots, s_{R}\right) \in S(r)\right\}
$$

$2 \Omega$ is determined by $\tau$ and the matrix $B$.

${ }^{3}$ Unlike $T(r)$ which is the upper bound for all $n, T_{A}(r)$ is the upper bound only in the asymptotic case. 
where

$$
S(r)=\left\{\begin{array}{ll}
\left(s_{1}, \ldots, s_{R}\right): & \\
\sum_{\omega \in \Omega} d_{i \omega} s_{\omega} \leq p_{i} r, & i=1,2, \ldots N, \\
s_{\omega} \geq 0, & \omega \in \Omega, \\
\sum_{\omega \in \Omega_{U}(\eta)} s_{\omega}=\sum_{\omega \in \Omega_{L}(\eta)} s_{\omega}, & \eta \in \tau, \\
\sum_{\omega \in \Omega} s_{\omega}=1, &
\end{array}\right\} .
$$

(Here, $s_{1}, s_{2}, \ldots$ are variables in the linear program.)

Note that $T_{A}(r)$ can be determined by computing a linear program with $R$ nonnegative variables and $N+1+|\tau|$ constraints. We denote this linear program as LP3.

Next, we describe the computation of the network capacity, $r_{0}^{A}$ in presence of both cochannel and adjacent channel use constraints. Recall that network capacity $r_{0}^{A}$ has the property that there exists a channel assignment algorithm which achieves arbitrarily low blocking probabilities, if the number of available channels is sufficiently large and the offered traffic $r$ is less than or equal to $r_{0}^{A}$. For $r>r_{0}^{A}$, no channel assignment algorithm can produce zero blocking for any number of channels. Now, [2] shows that

$r_{0}^{A}=\max r:\left\{\begin{array}{ll}\sum_{\omega \in \Omega} d_{i \omega} s_{\omega} \geq p_{i} r, & i=1,2, \ldots N \\ s_{\omega} \geq 0, & \omega \in \Omega \\ \sum_{\omega \in \Omega_{U}(\eta)} s_{\omega}=\sum_{\omega \in \Omega_{L}(\eta)} s_{\omega}, & \eta \in \tau \\ \sum_{\omega \in \Omega} s_{\omega}=1 . & \end{array}\right\}$.

Note that $r_{0}^{A}$ can be determined by computing a linear program with $R+1$ nonnegative variables and $N+|\tau|+1$ constraints. We denote this linear program as LP4.

In general, both $R$ and $|\tau|$ are very large. When cochannel reuse constraints are modeled by a hypergraph and adjacent channels not used in the same cell $(d=0), R \sim 10,|\tau| \sim 10$ for a system with three cells (refer Fig. 1), $R \sim 100,|\tau| \sim 10$ for a system with seven cells (refer Fig. 2), $R \sim 10^{6},|\tau| \sim 10^{3}$ for a system with 19 cells (refer Fig. 3) and $R \sim 10^{9},|\tau| \sim 10^{6}$ for a system with 37 cells (refer Fig. 4). Thus, both these linear programs are computationally intractable for systems of reasonable size.

We will not use LP3 and LP4 any further in this paper except for computing the exact values of $r_{0}^{A}$ and $T_{A}(r)$ for comparison with the corresponding approximations. In Sections IV and $\mathrm{V}$, we derive lower bounds on $r_{0}^{A}$ and $T_{A}(r)$, which we denote by $r_{0}^{A L}$ and $T_{A L}(r)$, respectively, using the solutions to the linear programs LP1 and LP2 for computing $T(r)$ and $r_{0}$ and a further graph theoretic approach. These lower bounds are computationally much simpler as $M \ll R$ and $N \ll|\tau| \ll R$. In general, $T_{A L}(r)$ and $r_{0}^{A L}$ are quite close to $T_{A}(r)$ and $r_{0}^{A}$ respectively. Note that upper bounds on $T_{A}(r)$ and $r_{0}^{A}$ are already known in the form of $T(r)$ and $r_{0}$ respectively. We first present some heuristics for designing fixed channel assignment algorithms with a minimum number of channels satisfying both

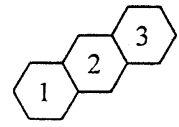

Fig. 1. A system with three cells.

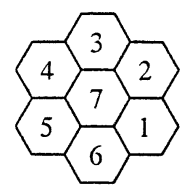

Fig. 2. A system with seven cells.

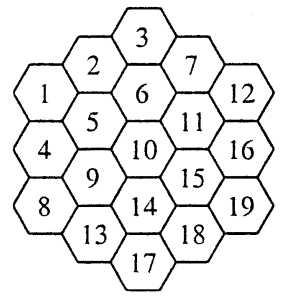

Fig. 3. A system with 19 cells.

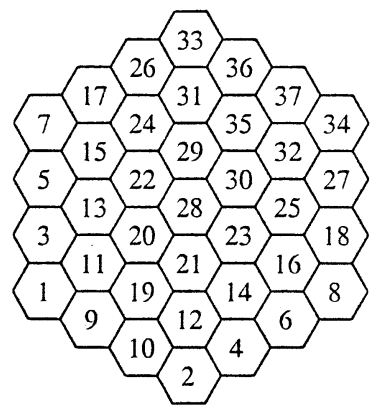

Fig. 4. A system with 37 cells.

cochannel and adjacent channel use constraints in the next section (Section III). The design strategy will introduce a graph theoretic approach which will be used subsequently to approximate $r_{0}^{A}$ and $T_{A}(r)$.

\section{HEURISTIC APPROACH FOR THE DETERMINATION OF THE NuMBER OF CHANNELS REQUIRED FOR A GIVEN FIXED CHANNEL ALlOCATION}

A channel allocation algorithm in which groups of channels are allocated to cells a priori and a cell accepts a requested call only if it has a free channel among those allocated to it is known as a fixed channel allocation algorithm. Though much more sophisticated algorithms, e.g., dynamic channel allocation schemes, have been devised which sometimes out perform the fixed channel allocation schemes, fixed channel allocation algorithms are still the ones in actual use because they are easy to implement. For a fixed channel allocation scheme, the number of channels required for cell $i, n_{i}$ can be determined from the traffic offered to cell $i$ and the acceptable blocking probability, using Erlang-B formula. Given the channel requirements for each cell, we may be interested in $n_{\text {min }}$, the minimum number of channels necessary to make the allocation, satisfying the cochannel reuse and the adjacent channel use constraints 
imposed by the transmission quality requirements. This will be less than $\sum_{i=1}^{N} n_{i}$ in general because of channel reuse. We have not found any efficient algorithm for this purpose in the literature when channel allocation needs to satisfy both cochannel and adjacent channel use constraints. We suggest a heuristic approach here.

We first describe the concept of graphs $F(\tilde{X}), F^{\prime}(\tilde{X})$ induced by a $M$-tuple $\tilde{X}=\left(X_{1}, X_{2}, \ldots, X_{M}\right)$. $\tilde{X}$ be any $M$-tuple such that $X_{j}$ is a nonnegative rational number and $M$ is the number of maximal independent sets of the hypergraph modeling the cochannel reuse constraints of the cellular system. Let $X_{k_{1}}, X_{k_{2}}, \ldots, X_{k_{V}}$ be the ones with nonzero values. Let $X_{k_{1}}: X_{k_{2}}: \cdots: X_{k_{V}}=m_{k_{1}}: m_{k_{2}}: \cdots: m_{k_{V}}$, where $m_{k_{1}}, m_{k_{2}}, \ldots, m_{k_{V}}$ are relatively prime positive integers. If $X_{j}=0, m_{j}=0$. It is convenient to think of $m_{k_{l}}$ as being (proportional to) the number of channels that are to be assigned to maximal independent set $X_{k_{l}}$. Form a graph $F(\tilde{X})=\left(V_{F}, E_{F}\right)^{4}$ as follows. $V_{F}=\left\{v_{k_{1}}, v_{k_{2}}, \ldots, v_{k_{V}}\right\}$. Vertices $v_{k_{l}}$ and $v_{k_{t}}$ are joined by an edge iff $b_{i j}=1$ for each cell $i$ in the $k_{l}$ th maximal independent set and each cell $j$ in the $k_{t}$ th maximal independent set of the hypergraph modeling the cochannel reuse constraints for the cellular system, i.e., if any two cells such that one is in the $k_{l}$ th maximal independent set and the other in the $k_{t}$ th maximal independent set can use adjacent channels simultaneously. Next, form a graph $F^{\prime}(\tilde{X})=\left(V_{F^{\prime}}, E_{F^{\prime}}\right) . V_{F^{\prime}}=\left\{v_{k_{1} 1}, \ldots, v_{k_{1} m_{k_{1}}}, v_{k_{2} 1}\right.$, $\left.\ldots, v_{k_{2} m_{k_{2}}}, \ldots, v_{k_{V} 1}, \ldots, v_{k_{V} m_{k_{V}}}\right\}$.

Vertices $v_{k_{l} a}$ and $v_{k_{t} b}$ have an edge between them iff the vertices $v_{k_{l}}$ and $v_{k_{t}}$ have an edge between them in $F(\tilde{X})$. In effect, each vertex in $F(\tilde{X})$ represents a maximal independent set (which is to be assigned at least one channel) and to obtain $F^{\prime}(\tilde{X})$ each vertex in $F(\tilde{X})$ is replaced by $m$ vertices where $m$ is (proportional to) the number of channels to be assigned to the maximal independent set represented by that vertex. We say that $F(\tilde{X})$ and $F^{\prime}(\tilde{X})$ have been induced by the $M$-tuple $\tilde{X}=\left(X_{1}, X_{2}, \ldots, X_{M}\right)$.

Theorem 1: Let $F(\tilde{X})$ and $F^{\prime}(\tilde{X})$ be the graphs induced by some $M$-tuple $\tilde{X}=\left(X_{1}, X_{2}, \ldots, X_{M}\right)$. Let $F^{\prime}(\tilde{X})$ become Hamiltonian ${ }^{5}$ upon the addition of some $p$ edges. Let $q\left(p+\sum_{j=1}^{M} m_{j}\right)-1_{\{p q>0\}^{6}}{ }^{\text {channels be available. Then there }}$ exists a fixed channel allocation algorithm which allocates $q \sum_{j=1}^{M} m_{j} a_{i j}$ channels to the $i$ th cell, for $1 \leq i \leq N$, for any nonnegative integer $q$.

Note that the proof exhibits a channel allocation algorithm which attains the purpose.

Proof: Let $q\left(p+\sum_{j=1}^{M} m_{j}\right)-1_{\{p q>0\}}$ channels be available. If $q=0$ the lemma is trivially true. Let $q>0$. We say that we allocate a channel to a maximal independent set when we allocate the channel to each cell in the maximal independent set. First, we show that it is possible to allocate $q m_{j}$ channels to the $j$ th maximal independent set without violating the

\footnotetext{
${ }^{4}$ In our notation in any graph $G=(V, E), V$ is the vertex set and $E$ is the edge set.

${ }^{5} \mathrm{~A}$ cycle in a graph is a sequence of vertices such that consecutive vertices in the cycle have an edge between them and no vertex in the sequence occurs more than once except the first (or the last), which occurs twice, once in the first position and again in the last position. A graph is Hamiltonian iff it has a cycle consisting of all vertices. Such a cycle is known as a Hamilton cycle.

${ }^{6} 1_{\{p q>0\}}=1$ iff $p q>0$ and $1_{\{p q>0\}}=0$, otherwise.
}

cochannel reuse and adjacent channel use constraints. Let the Hamilton cycle of vertices of $F^{\prime}$ formed upon the addition of $p$ edges to $F^{\prime}$ be $v_{i_{0}}, j_{0}, v_{i_{1}}, j_{1}, \ldots, v_{i_{U-1}, j_{U-1}}, v_{i_{0}}, j_{0}$. Note that $U=\sum_{j=1}^{M} m_{j}$. Some of the consecutive vertices in this cycle are linked by an edge in $F^{\prime}$ and some by an added edge if $p>0$. If $p>0$, without loss of generality, $v_{i_{U-1}, j_{U-1}}$ and $v_{i_{0} j_{0}}$ are joined by an added edge. Allocate channels to the maximal independent sets as per the following algorithm.

1) $a=0, b=0, c=1$.

2) Give channel numbered $b$ to the $i_{a}$ th maximal independent set.

3) If $a=U-1, c \rightarrow c+1$. If $c>q$ stop.

4) If $v_{i_{a}, j_{a}}$ and $v_{i_{(a+1) \bmod U} j_{(a+1) \bmod U}}$ are linked by an edge in $F^{\prime}$, then $b \rightarrow b+1$; else $b \rightarrow b+2 . a \rightarrow(a+1) \bmod U$. Go to step 2).

Observe that we are moving along the Hamilton cycle $q$ times and each time the vertex $v_{i_{l} j_{l}}$ is encountered the $i_{l}$ th maximal independent set is given a channel. Since we are allocating different channels to different maximal independent sets, the cochannel reuse constraints are satisfied. Two cells $i$ and $j$ can get adjacent channels only if $i$ is in a maximal independent set $k_{1}$ and $j$ in $k_{2}$ such that the vertices corresponding to the two maximal independent sets $k_{1}$ and $k_{2}$ are linked by an edge in $F^{\prime}$ and, hence, in $F$ ( $v_{d e}$ and $v_{f g}$ have an edge between them in $F^{\prime}$ iff $v_{d}$ and $v_{f}$ have an edge between them in $F$ ). This means that any cell $i$ in the maximal independent set $k_{1}$ and $j$ in $k_{2}$ are permitted to use adjacent channels simultaneously. Thus, the adjacent channel use constraints are satisfied. The vertex $v_{i_{l} j_{l}}$ is encountered $q$ times while traversing the Hamilton cycle $q$ times giving $q$ different channels to the $i_{l}$ th maximal independent set, for each $i_{l}$ such that $X_{i_{l}}>0$ and $j_{l} \in\left\{1,2, \ldots, m_{i_{l}}\right\}$. Thus, each of the following vertices $v_{i_{l} 1}, v_{i_{l}}, \ldots, v_{i_{l}}, m_{i_{l}}$ give $q$ channels to the $i_{l}$ th maximal independent set, giving it $q m_{i_{l}}$ channels, if $X_{i_{l}}>0$. If $X_{j}=0$, the $j$ th maximal independent set gets $q m_{j}=0$ channels. If $p=0$ no channel has been skipped. ${ }^{7}$ If $p>0, p-1$ channels are skipped the last time the Hamilton cycle is traversed and $p$ channels are skipped each of the other $q-1$ times the Hamilton cycle is traversed, because of the $p$ added edges. Thus, $q\left(p+\sum_{j=1}^{M} m_{j}\right)-1_{\{p q>0\}}$ channels have been used to allocate $q m_{j}$ channels to the $j$ th maximal independent set satisfying the cochannel reuse and adjacent channel use constraints. This fixed channel allocation algorithm allocates $q \sum_{j=1}^{M} m_{j} a_{i j}$ channels to the $i$ th cell.

Consider the following integer linear program:

$$
\left.\begin{array}{ll}
\operatorname{Minimize} \sum_{j=1}^{M} W_{j} & \\
\sum_{j=1}^{M} W_{j} a_{i j} \geq n_{i}, & i=1,2, \ldots N \\
W_{j} \geq 0, & j=1,2, \ldots, M, \\
& W_{j} \text { integer. }
\end{array}\right\}
$$

Let $n$ be the optimal value of the objective function. Consider graphs $G=F\left(W_{1}^{O}, \ldots, W_{M}^{O}\right)$ and $G^{\prime}=F\left(W_{1}^{O}, \ldots, W_{M}^{O}\right)^{\prime}$

\footnotetext{
${ }^{7}$ A channel is said to be "skipped" if it is not allocated to any maximal independent set.
} 
induced by the optimal solution $\left(W_{1}^{O}, \ldots, W_{M}^{O}\right)$. The following Corollary follows from Theorem 1.

Corollary 1: Let $G^{\prime}$ become Hamiltonian on the addition of $p$ edges. Then $n+(q p-1)^{+}$channels are sufficient for the fixed channel allocation algorithm which allocates $n_{i}$ channels to the $i$ th cell, for $1 \leq i \leq N$, where $q=$ g.c.d. of nonzero $W_{j}^{O}$ s, if there exists at least one nonzero $W_{j}^{O}$ and $q=0$ otherwise.

We summarize the heuristic approach as follows.

1) Compute the ILP. Let $n$ be the optimal value of the objective function. Let $\left(W_{1}^{O}, W_{2}^{O}, \ldots, W_{M}^{O}\right)$ be the optimal solution. $q=$ g.c.d. of nonzero $W_{i}^{O}$, if there exists at least one nonzero $W_{j}^{O}$ and $q=0$, otherwise.

2) Form $G$ and $G^{\prime}$ from the optimal solution as discussed above, i.e., $G=F\left(W_{1}^{O}, \ldots, W_{M}^{O}\right)$ and $G^{\prime}=F\left(W_{1}^{O}, \ldots, W_{M}^{O}\right)^{\prime}$

3) Compute the minimum number of edges $p_{\min }$ required to make $G^{\prime}$ Hamiltonian or an approximation to it, say $p$. We discuss how to compute of $p_{\min }$ in Section IV.

4) Use $n+(p q-1)^{+}$channels to make the fixed channel allocation. The closer $p$ is to $p_{\min }$, fewer the number of additional edges required to make $G^{\prime}$ Hamiltonian and lesser the number of channels used.

5) The algorithm for making the required fixed channel allocation using $n+(p q-1)^{+}$channels follows from the proof of Theorem 1. Find the channels allocated to the $j$ th maximal independent set by the algorithm described in the proof to allocate $W_{j}^{O}=q m_{j}$ channels to the $j$ th maximal independent set. All the channels allocated to the $j$ th maximal independent set are allocated to each of the cells in the $j$ th maximal independent set. This gives the actual channel allocation to the cells.

If $p q$ is small compared to $n$, then the number of channels obtained from our heuristic will be close to the minimum since $n$ channels are necessary for making the fixed channel allocation even while satisfying only the cochannel reuse constraints. We illustrate the actual channel allocation to the cells in the following examples.

Example III.1: Consider the system with seven cells shown in Fig. 2. Let the cochannel reuse constraint be that the maximum interference should not exceed 0.15 units. A channel may be used simultaneously in any number of cells, as long as the total interference does not exceed 0.15. Assume that the cell radius is $1 / \sqrt{3}$. Distance between adjacent cells is one unit, and distance between nonadjacent two-hop away cells like one and three is $\sqrt{3}$. Assume that the interference produced in cell $u$ by the simultaneous use of a channel in cell $v$ is $d(u, v)^{-4}$. Thus, adjacent cells cannot use the same channel simultaneously (interference will be 1). No combination of three or more cells can use a channel simultaneously, e.g., the total interference produced in cell 3 if cells $1,3,5$ use the same channel simultaneously is $2 / 9\left(d(1,3)^{-4}+d(3,5)^{-4}=2 / 9\right)$. However, cells 1 and 3 can simultaneously use the same channel, when cell 5 is not using it. The hypergraph modeling this cochannel reuse constraint has ten maximal independent sets: $\{1,3\},\{1,4\}$, $\{1,5\},\{2,4\},\{2,5\},\{2,6\},\{3,5\},\{3,6\},\{4,6\},\{7\}$. We need to allocate four channels to each cell. Thus, $n_{i}=4$, for

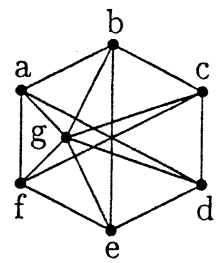

(a) (b)

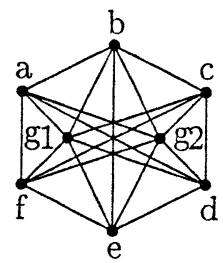

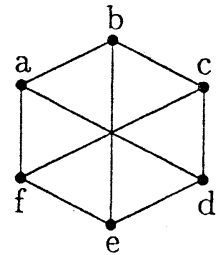

(c)
Fig. 5. The graphs for the system with seven cells when use of adjacent channel is prohibited in the same cell: (a) shows the graph $G^{\prime}$, (b) the graph $G$, and (c) the basic interference graph.

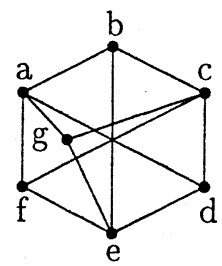

(a)

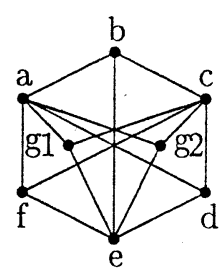

(b)
Fig. 6. The graphs for the system with seven cells when adjacent channel use is prohibited in the same cell, and also cell seven cannot use a channel if its adjacent channel is being used in cell 2,4 , or 6 . (a) shows the graph $G$ and (b) the graph $G^{\prime}$.

$1 \leq i \leq 7$. The ILP gives nonzero values to only the following maximal independent sets: $a \rightarrow\{1,3\}, b \rightarrow\{2,4\}, c \rightarrow$ $\{3,5\}, d \rightarrow\{4,6\}, e \rightarrow\{1,5\}, f \rightarrow\{2,6\}, g \rightarrow\{7\}$. $a=b=c=d=e=f=2, g=4, n=16, q=2$, $m_{a}=m_{b}=m_{c}=m_{d}=m_{e}=m_{f}=1$ and $m_{g}=2$. We shall consider two different adjacent channel use constraints.

Case 1) Adjacent channels cannot be used simultaneously only in the same cell. The graphs $G$ and $G^{\prime}$ have been shown in Fig. 5. $G^{\prime}$ is Hamiltonian. Thus, $p_{\min }=0$ and 16 channels are sufficient. $a g 1 b c d g 2 e f$ $a$ is a Hamilton cycle in $G^{\prime}$. Proceeding as per the algorithm in Theorem 1 with $q=2, a$ gets channels $1,9, g 1$ gets $2,10, b$ gets $3,11, c$ gets $4,12, d$ gets $5,13, g 2$ gets $6,14, e$ gets 7,15 and $f$ gets 8,16 . Thus, cell 1 gets channels $\{1,7,9,15\}$, cell 2 gets $\{3,8,11,16\}$, cell 3 gets $\{1,4,9,12\}$, cell 4 gets $\{3,5,11,13\}$, cell 5 gets $\{4,7,12,15\}$, cell 6 gets $\{5,8,13,16\}$, and cell 7 gets $\{2,6,10,14\}$.

Case 2) Again assume that adjacent channels cannot be used simultaneously in the same cell and also that cell 7 cannot use a channel if its adjacent channel is being used in either cell 2, 4, or 6. Refer to Fig. 6(a) and (b) for $G$ and $G^{\prime}$, respectively. $G^{\prime}$ is not Hamiltonian. However, it becomes Hamiltonian when edges are added between the pairs of vertices $(g 1, b)$ and $(d, g 2)$. Thus, $p_{\min } \leq 2$ and 19 channels are sufficient. $b c d g 2$ e $f a g 1 b$ is a Hamilton cycle in $G^{\prime}$ with the added edges. Proceeding as per the algorithm in Theorem 1 with $q=2, b$ gets channels $1,11, c$ gets $2,12, d$ gets $3,13, g 2$ gets 5 , $15, e$ gets $6,16, f$ gets $7,17, a$ gets $8,18 g 1$ gets 9 , 19 . Thus, cell 1 gets channels $\{6,8,16,18\}$, cell 2 
gets $\{1,7,11,17\}$, cell 3 gets $\{2,8,12,18\}$, cell 4 gets $\{1,3,11,13\}$, cell 5 gets $\{2,6,12,16\}$, cell 6 gets $\{3,7,13,17\}$, and cell 7 gets $\{5,9,15,19\}$.

For large systems the ILP is difficult to evaluate. The integer constraints in the ILP may be relaxed to get an approximate solution. Thus, $n_{\text {approx }}=\sum_{j=1}^{M}\left\lceil W_{j}^{O}\right\rceil$ can then be taken as an approximation to $n$ and $\left(\left\lceil W_{1}^{O}\right\rceil,\left\lceil W_{2}^{O}\right\rceil, \ldots,\left\lceil W_{M}^{O}\right\rceil\right)$ can be used in place of the optimal solution to the ILP. Clearly $n_{\text {approx }}+(p q-1)^{+}$channels, with $p$ obtained from the corresponding $G$ and $G^{\prime}$ and $q$ from the $\left(\left\lceil W_{1}^{O}\right\rceil\right.$, $\left.\left\lceil W_{2}^{O}\right\rceil, \ldots,\left\lceil W_{M}^{O}\right\rceil\right)$, is sufficient for the required fixed channel allocation. In general $n_{\text {approx }}$ is a good approximation to $n$, the optimal value of the objective function of the ILP.

\section{ApProximation to $r_{0}^{A}$}

We will present an approximation strategy for $r_{0}^{A}$. We use a two-step procedure here. We start with the optimal solution of LP2 which satisfies the cochannel constraints. Next, we modify this optimal solution with the objective of satisfying the adjacent channel constraints, using a graph theoretic technique similar to that of Section III. We describe the approach in details next.

Let $G$ and $G^{\prime}$ be the graphs induced by a rational $M$-tuple $\left(X_{1}^{O}, X_{2}^{O}, \ldots, X_{M}^{O}\right)$, which forms an optimal solution of LP2. (Clearly any extreme point of the feasible region corresponds to rational values of $X_{1}^{O}, X_{2}^{O}, \ldots, X_{M}^{O}$ because the constraints involve rational numbers only. We know at least one of the extreme points gives the optimal solution, if there exists an optimal solution [8]. LP2 always has an optimal solution [4]. Thus, there always exists a rational $M$-tuple $\left(X_{1}^{O}, X_{2}^{O}, \ldots, X_{M}^{O}\right)$ which forms an optimal solution of LP2.) $\gamma_{G}$ (the number of vertices in $G)=V \leq N$ and $\gamma_{G^{\prime}}=\sum_{j=1}^{M} m_{j}$.

Theorem 2: Let $G^{\prime}$ become Hamiltonian upon the addition of some $p$ edges. Then $r_{0}^{A} \geq r_{0} /\left(1+p / \sum_{j=1}^{M} m_{j}\right)$.

Proof: Let there be $n$ channels. Then $n=q(p+$ $\left.\sum_{j=1}^{M} m_{j}\right)+t$, where $0 \leq t<\left(p+\sum_{j=1}^{M} m_{j}\right)$ and $q$ is a nonnegative integer (Euclidean division theorem). By Theorem 1 we have a fixed channel allocation algorithm that allocates $q \sum_{j=1}^{M} m_{j} a_{i j}$ channels to the $i$ th cell, for $1 \leq i \leq N$. Using the ATP and the independence of offered traffic from cell to cell it can be shown that this fixed channel allocation algorithm carries a traffic intensity of $\min \left(p_{i} r,\left(\sum_{j=1}^{M} m_{j} a_{i j} / p+\sum_{j=1}^{M} m_{j}\right)\right)$ in the asymptotic limit $(n \rightarrow \infty)$ in cell $i$ (refer to the Appendix for the proof).

Let $r \leq r_{0} /\left(1+p / \sum_{j=1}^{M} m_{j}\right)$. Then

$$
\begin{aligned}
p_{i} r & \leq p_{i} r_{0} /\left(1+p / \sum_{j=1}^{M} m_{j}\right) \\
& \leq \frac{\sum_{j=1}^{M} X_{j}^{O} a_{i j}}{\left(1+p / \sum_{j=1}^{M} m_{j}\right)}
\end{aligned}
$$

from the third set of inequalities in LP2

$$
\begin{gathered}
=\frac{\frac{\sum_{j=1}^{M} m_{j} a_{i j}}{\sum_{k=1}^{M} m_{k}}}{\left(1+p / \sum_{j=1}^{M} m_{j}\right)} \\
\text { since } X_{j}^{O}=\frac{m_{j}}{\sum_{k=1}^{M} m_{k}} \\
=\frac{\sum_{j=1}^{M} m_{j} a_{i j}}{\left(p+\sum_{j=1}^{M} m_{j}\right)} .
\end{gathered}
$$

Thus, in the asymptotic limit the carried traffic intensity in the $i$ th cell is $\lim _{n \rightarrow \infty} C\left(p_{i} r n, q \sum_{j=1}^{M} m_{j} a_{i j}\right) / n$ $=\min \left(p_{i} r, \sum_{j=1}^{M} m_{j} a_{i j} /\left(p+\sum_{j=1}^{M} m_{j}\right)\right)=p_{i} r$. Thus, the blocking probability is zero in the asymptotic limit for this fixed channel allocation algorithm if $r \leq r_{0} /\left(1+p / \sum_{j=1}^{M} m_{j}\right)$. Thus, $r_{0}^{A} \geq r_{0} /\left(1+p / \sum_{j=1}^{M} m_{j}\right)$ since $r_{0}^{A}$ is the largest value of $r$ for which a channel assignment algorithm that has asymptotically zero blocking exists.

Let $r_{0}^{A L}=r_{0} /\left(1+p / \sum_{j=1}^{M} m_{j}\right)$. Clearly the lower bound is the tightest if $p=p_{\min }$, where $p_{\min }$ is the minimum number of edges which must be added to $G^{\prime}$ to make it Hamiltonian. In general, the problem of finding $p_{\min }$ is NP-complete [9] but $p_{\text {min }}$ can be found easily in the following special cases.

1) $G$ is a complete ${ }^{8}$ graph. Let $G^{\prime}$ have $\gamma_{G^{\prime}}$ vertices. Then

$$
p_{\min }= \begin{cases}\left(\max _{j} m_{j}-\sum_{\substack{j=1 \\ j \neq \operatorname{argmax}_{k} m_{k}}}^{M} m_{j}\right)^{+}, & \text {if } \gamma_{G^{\prime}} \geq 3, \\ 0, & \text { if } \gamma_{G^{\prime}}=2 \\ 1, & \text { if } \gamma_{G^{\prime}}=1 .\end{cases}
$$

The proof goes as follows. Let $\gamma_{G^{\prime}} \geq 3$, and let $\max _{j} m_{j} \leq \sum_{j=1 j \neq \arg \max _{k} m_{k}}^{M} m_{j}$. Let $v_{j k}$ be a vertex in $G^{\prime} \cdot \gamma_{G^{\prime}}=\sum_{i=1}^{M} m_{i}$. The degree of a vertex $v$ in a graph $G$ [denoted by $\left.d_{G}(v)\right]$ is the number of edges incident on $v$. $d_{G^{\prime}}\left(v_{j k}\right)=\sum_{i=1 i \neq j}^{M} m_{i}=\gamma_{G^{\prime}}-m_{j}$

$$
\begin{aligned}
\max _{i} m_{i} & \leq \sum_{\substack{i=1 \\
i \neq \arg _{\max _{l}} m_{l}}}^{M} m_{i} \\
2 \max _{i} m_{i} & \leq \sum_{\substack{i=1 \\
i \neq \arg \max _{l} m_{l}}}^{M} m_{i}+\max _{i} m_{i} \\
2 \max _{i} m_{i} & \leq \sum_{i=1}^{M} m_{i}
\end{aligned}
$$

${ }^{8} \mathrm{~A}$ graph is simple if no edge joins a vertex to itself. A graph is complete if it is simple and any two distinct vertices in the graph has an edge between them [10]. 
TABLE II

A Comparison of the Complexities of Deora's Method [2] And Our Method in the Case Where Adjacent Channel

Use in the SAME Cell Alone is Prohibited

\begin{tabular}{|c|c|c|c|c|c|c|c|c|c|}
\hline \multirow{4}{*}{$\begin{array}{l}\text { No. of } \\
\text { cells }\end{array}$} & \multirow{4}{*}{$\begin{array}{l}\text { Cochannel } \\
\text { Interf. } \\
\text { Threshold }\end{array}$} & \multirow{2}{*}{\multicolumn{2}{|c|}{$\frac{\text { Deora's method[2] }}{\text { Number of }}$}} & \multicolumn{6}{|c|}{ Our method } \\
\hline & & & & \multicolumn{2}{|c|}{ Number of } & \multirow{2}{*}{\multicolumn{4}{|c|}{$\begin{array}{l}\text { Number of } \\
\text { vertices in }\end{array}$}} \\
\hline & & \multirow[t]{2}{*}{ vars. } & \multirow[t]{2}{*}{ constrs. } & \multirow[t]{2}{*}{ vars } & \multirow[t]{2}{*}{ constrs } & & & & \\
\hline & & & & & & UT & NUT & UT & NUT \\
\hline 3 & $(0,1)$ & 17 & 9 & 3 & 4 & 2 & & 2 & \\
\hline 7 & 0.15 & 201 & 25 & 11 & 8 & 7 & & 8 & \\
\hline 19 & 0.1 & 53515 & 311 & 85 & 20 & 17 & 18 & 98 & 74 \\
\hline 19 & 0.2 & 344539 & 833 & 249 & 20 & 8 & 14 & 18 & 624998 \\
\hline 19 & 0.3 & 681543 & 1238 & 242 & 20 & 18 & 17 & 147 & 50 \\
\hline 19 & 0.375 & 802297 & 1373 & 188 & 20 & 17 & 11 & 49 & 500006 \\
\hline 19 & 0.4 & 824137 & 1397 & 194 & 20 & 18 & & 82 & \\
\hline 19 & 0.5 & 856699 & 1432 & 167 & 20 & 6 & & 10 & \\
\hline 19 & 0.6 & 867139 & 1444 & 146 & 20 & 9 & & 19 & \\
\hline 19 & $2 / 3$ & 867971 & 1445 & 140 & 20 & 3 & & 3 & \\
\hline 37 & 0.1 & 235796085 & 21310 & 4987 & 38 & 34 & & 1111 & \\
\hline
\end{tabular}

$$
\begin{aligned}
\max _{i} m_{i} & \leq \frac{\sum_{i=1}^{M} m_{i}}{2} \\
m_{j} & \leq \frac{\gamma_{G^{\prime}}}{2} \quad \text { for all } j \\
d_{G^{\prime}}\left(v_{j k}\right) & \geq \frac{\gamma_{G^{\prime}}}{2} .
\end{aligned}
$$

Thus, $G^{\prime}$ is Hamiltonian [10, p. 54, Th. 4.3]). Thus, $p_{\min }=0$ and the relation holds in this case.

Let $\max _{j} m_{j}>\sum_{j=1 j \neq \arg \max _{k} m_{k}}^{M} m_{j}$. Let $i=$ $\arg \max _{k} m_{k} . v_{i 1}, v_{i 2}, \ldots, v_{i m_{i}}$ must be part of the Hamilton cycle. Clearly $v_{i a}, v_{i b}$ do not have an edge between them in $G^{\prime}$, since $G$ is simple. Thus, an extra edge must be added between $v_{i a}$ and $v_{i b}$ if they are consecutive vertices in the cycle. There must be at least $m_{i}-\sum_{j=1 j \neq i}^{M} m_{j}$ pairs $v_{i a}$ and $v_{i b}$ such that the vertices of the pair occupy consecutive positions in the cycle. Thus, $\max _{j} m_{j}-\sum_{j=1}^{M} j \neq \arg \max _{k} m_{k} m_{j}$ edges must be added to make $G^{\prime}$ Hamiltonian. By placing one $v_{j k}$ for some $k$ and some $j \neq i$ between $v_{i l}$ and $v_{i l+1}$ till the $v_{j k}$ s are exhausted, exactly $m_{i}-\sum_{j=1}^{M} m_{j \neq i} m_{j}$ pairs $v_{i a}$ and $v_{i b}$ will be there such that the vertices of the pair occupy consecutive positions in the cycle. Thus, only $\max _{j} m_{j}-\sum_{j=1 j \neq \arg \max _{k} m_{k}}^{M} m_{j}$ edges need be added to make $G^{\prime}$ Hamiltonian. Thus, $p_{\min }=\max _{j} m_{j}-\sum_{j=1}^{M} j \neq \arg \max _{k} m_{k} m_{j}$ in this case.

Let $\gamma_{G^{\prime}}=1$. Since $G$ is simple, $G^{\prime}$ must be simple and, thus, one edge must be added to make $G^{\prime}$ Hamiltonian. Let $\gamma_{G^{\prime}}=2$. Note that $\gamma_{G} \leq \gamma_{G^{\prime}}$. If $\gamma_{G}=1$, and $V_{G}=v_{l}, m_{l}=1$. $\left(X_{l}^{O}>0, X_{i}^{O}=0\right.$, if $i \neq l, \sum_{i=1}^{M} X_{i}^{O}=1 \Rightarrow X_{l}^{O}=1$, $m_{l}=1$.) This means $\gamma_{G^{\prime}}=1$ which is a contradiction. Thus, $\gamma_{G}=2$. Let $V_{G^{\prime}}=\left\{v_{i 1}, v_{j 1}\right\}$. Since $G$ is complete, so is $G^{\prime}$. Thus, there is an edge between $v_{i 1}$ and $v_{j 1}$. As per our definition, $v_{i 1} v_{j 1} v_{i 1}$ is a Hamilton cycle. Thus, no edge need be added to $G^{\prime}$ to make it Hamiltonian. Thus, $p_{\min }=0$ if $\gamma_{G^{\prime}}=2$.

$G$ is complete for many systems. For example, $G$ is complete for a linear array of cells, where any two cells can use the same channel simultaneously iff they are separated by a distance $D \geq$ $u Z$, where $Z$ is the center to center distance between adjacent cells and the adjacent channel use constraint is that the same cell cannot use adjacent channels simultaneously and $p_{i}=p_{i+u}$ for all $i$. Thus, $p_{\min }$ can be computed easily for these systems.
TABLE III

THE LOWER BOUNDS ON CAPACITIES OBTAINED USING OUR METHOD IN the CASE Where AdJacent Channel USE in the SAME Cell ALONE IS PROHIBITED

\begin{tabular}{|r|l|l|l|r|r|r|r|}
\hline $\begin{array}{r}\text { No. of } \\
\text { cells }\end{array}$ & $\begin{array}{l}\text { Cochannel } \\
\text { Interf. } \\
\text { Threshold }\end{array}$ & UT $^{\text {AL }} / r_{0}$ & NUT & UT & NUT & UT & NUT \\
\hline 3 & $0.1)$ & 1.0 & & 1.50 & & 1.50 & \\
7 & 0.15 & 1.0 & & 1.75 & & 1.75 & \\
19 & 0.02 & 1.0 & 1.0 & 2.11 & 1.85 & 2.11 & 1.85 \\
19 & 0.1 & 1.0 & 1.0 & 3.10 & 2.56 & 3.10 & 2.56 \\
19 & 0.2 & 1.0 & 1.0 & 4.22 & 3.20 & 4.22 & 3.2 \\
19 & 0.3 & 1.0 & 1.0 & 4.65 & 3.84 & 4.65 & 3.84 \\
19 & 0.375 & 1.0 & 1.0 & 5.04 & 4 & 5.04 & 4 \\
19 & 0.4 & 0.99 & 1.0 & 5.04 & 4 & 5.10 & 4 \\
19 & 0.5 & 1.0 & 1.0 & 5.73 & 4 & 5.73 & 4 \\
19 & 0.6 & 1.0 & 1.0 & 6.00 & 4 & 6.00 & 4 \\
19 & $2 / 3$ & 1.0 & 1.0 & 6.33 & 4 & 6.33 & 4 \\
37 & 0.1 & 1.0 & & 5.13 & & 5.13 & \\
37 & 0.12 & 1.0 & & 5.69 & & 5.69 & \\
37 & 0.39 & 1.0 & & 9.25 & & 9.25 & \\
37 & 0.5 & 1.0 & & 9.25 & & 9.26 & \\
37 & 0.6 & 1.0 & & 10.74 & & 10.74 & \\
37 & 0.7 & 1.0 & & 10.96 & & 10.98 & \\
37 & 0.74 & 1.0 & & 12.33 & & 12.33 & \\
\hline
\end{tabular}

2) $G$ is completely disconnected, i.e., $G$ has no edge. Thus, $G^{\prime}$ is also completely disconnected. Thus, $p_{\min }=\gamma_{G^{\prime}}=\sum_{j=1}^{M} m_{j}$. We have found that $G$ is in general disconnected if adjacent channels cannot be used simultaneously in the same or adjacent cells.

3) There are certain sufficient conditions involving the degree sequence and the number of edges in $G^{\prime}$ [10], [11] for $G^{\prime}$ to be Hamiltonian. If these are satisfied, then $p_{\min }=0$. Often a good approximation to $p_{\min }$ can be found from these sufficiency conditions.

If none of the above cases apply, then we need to resort to other means to find $p_{\min }$. The problem of finding $p_{\min }$ can be reduced to a traveling salesman problem [10]. The traveling salesman problem is an NP-complete problem but there exist techniques which yield good approximations to the required result [9], [10].

We have used the following seemingly crude method which surprisingly gives small values of $p$ in a very short time for all the cases we have studied and whose results we shall present in Tables II and III. We first briefly describe our method. Finding a Hamilton cycle in $G^{\prime}$ is equivalent to finding a closure-possible 
walk ${ }^{9}$ in $G\left(\gamma_{G} \leq N\right)$ traversing vertex $v_{j} m_{j}$ times. The length of the walk must be $\gamma_{G^{\prime}}$. We have observed that a simple branch and backtrack ${ }^{10}$ technique gives a walk of length, say $T$, very fast, where $T$ is fairly close to $\gamma_{G^{\prime}}$. Moreover this walk traverses no vertex $v_{j}$ more than $m_{j}$ times but after the length of the walk increases to $T$ any further increase takes place very slowly. We stop the branch and backtrack process after a walk, which is $T$ vertices long is obtained. If this walk traverses the vertex $v_{j} n_{j}$ times, we try to insert $m_{j}-n_{j} v_{j}$ s in the walk that has already been obtained. If we cannot do so, we add extra edges, which increase the value of $p$ we can get. The number $T$ is decided upon after a few observations. We illustrate the branch and backtrack procedure by the example that follows.

Example IV.1: Let the graph $G$ consist of $W$ vertices numbered $1,2, \ldots, W$ where $W$ is quite large. The edges in $G$ are as follows: $i$ and $i+1$ are joined by an edge for all $i<W-1$. $W$ has an edge with 1 and $2 . W-1$ has an edge with 1 . There is no other edge in G. $m_{i}=1$ for all $i$. The only closure-possible walk covering each vertex once is $1 W 23 \cdots W-1$. (We consider cyclic shifts such as $1 W 23 \cdots W-1$ and $W 23 \cdots W-1$ 1 as being equivalent.) However, the branch and backtrack procedure may move along the branch $123 \cdots W-1$. Thus, a walk of length $W-1$ is obtained very fast but then on, the process backtracks in order to insert $W$ and this may take a long time. If the procedure is terminated when the walk of length $W-1$ is obtained, then $W$ can be very easily inserted between 1 and 2 and, thus, a closure-possible walk is obtained and $p=0$. If $W$ could not be inserted i.e., if it did not have edges joining it to 1 and 2, then two edges could have been added, one joining $W$ to 1 and the other to 2, and a closure-possible walk could have been obtained, giving $p=2$. This value of $p$ is small, particularly if $W$ is large and, thus, we get a good approximation to $p_{\min }$ in a very short time.

If the graph $G^{\prime}$ does not give a small value of $p$ fast with the above method with a particular optimal solution, the graph $G^{\prime}$ obtained from some other rational optimal solution may be tried. This often helps when there are multiple optimal solutions (degeneracy).

We now present our results for systems with 3, 7, 19, 37 regular hexagonal cells (refer to Figs. 1-4). We have assumed that the cochannel reuse in the system is constrained by the maximum tolerable interference. (Any two calls in the system using the same channel simultaneously interfere with each other. The interference diminishes with increase in distance between the callers.) The assumed model of interference is as follows:

- interference produced in cell $u$ due to the usage of the same channel in cell $v$ equals $d(u, v)^{-4}$, where $d(u, v)$ is the center-to-center distance between cells $u$ and $v$;

- total interference produced in cell $u=$ interference produced by all other cells using the same channel $=\sum_{v \in C(u)} d(u, v)^{-4}$, where $C(u)$ is the set of cells

\footnotetext{
${ }^{9} \mathrm{~A}$ walk in a graph is a sequence of vertices such that the consecutive vertices in the sequence have an edge between them. A walk is closure-possible if the first and the last vertices have an edge between them.

${ }^{10}$ This procedure traverses along the graph without visiting any vertex $v_{j}$ more than $m_{j}$ times as long as it can, and when it cannot it backtracks along the traced path, until it can proceed along some branch which has not yet been visited.
}

using the same channel as $u$, barring $u$; an additive model of interference is thus assumed;

- the cell radius is assumed to be $1 / \sqrt{3}$ or equivalently the distance between adjacent cells is taken to be 1 ;

- let the requisite transmission quality be that the maximum interference must be less than or equal to some given threshold; this limits cochannel reuse.

This model for interference is the same as that used in [4]. The cochannel reuse constraints have been modeled by a hypergraph. We illustrate the computation of the approximation to $r_{0}^{A}$ by the following.

Example IV.2: Consider the system with seven cells shown in Fig. 2 described in Example III.1. The same model for interference is assumed as in Section III. The cochannel reuse constraints, and hence the maximal independent sets of the hypergraph modeling the cochannel reuse constraints, remain the same as in Example III.1. LP2 gives nonzero values to only the following maximal independent sets: $a \rightarrow\{1,3\}, b \rightarrow\{2,4\}$, $c \rightarrow\{3,5\}, d \rightarrow\{4,6\}, e \rightarrow\{1,5\}, f \rightarrow\{2,6\}, g \rightarrow\{7\}$. $a=b=c=d=e=f=1 / 8, g=1 / 4, m_{a}=m_{b}=m_{c}=$ $m_{d}=m_{e}=m_{f}=1$, and $m_{g}=2$. We shall consider two different adjacent channel use constraints as in Example III.1.

Case 1) Adjacent channels cannot be used simultaneously only in the same cell. Thus, $b_{i i}=2$ for all $i$ and $b_{i j}=$ 1 , if $i \neq j$. The graphs $G$ and $G^{\prime}$ have been shown in Fig. 5(a) and (b), respectively. $G^{\prime}$ is Hamiltonian. Thus, $p_{\min }=0$ and $r_{0}^{A L}=r_{0}=1.75$. Clearly, $r_{0}^{A L}=r_{0}^{A}$ here. $a g 1 b c d g 2$ e $f a$ is a Hamilton cycle in $G^{\prime}$.

Case 2) Assume adjacent channels cannot be used simultaneously in the same cell and also that cell seven cannot use a channel if its adjacent channel is being used in either cell 2,4 , or 6 . Thus

$$
b_{i j}=\left\{\begin{aligned}
2, & \text { if } i=j, \\
& \text { or }(i, j) \in\{(2,7),(4,7),(6,7), \\
& (7,2),(7,4),(7,6)\}, \\
1, & \text { otherwise. }
\end{aligned}\right.
$$

Refer to Fig. 6(a) and (b) for $G$ and $G^{\prime}$, respectively. $G^{\prime}$ is not Hamiltonian. However, it becomes Hamiltonian when edges are added between $g 1, b$ and $d, g 2$. Thus, $p_{\min } \leq 2$ and $r_{0}^{A L}=0.8 r_{0}=1.4$. Using $r_{0}^{A L} \leq r_{0}^{A} \leq r_{0}, 1.4 \leq r_{0}^{A} \leq 1.75 . b c d g 2$ e f $a g 1 b$ is a Hamilton cycle in $G^{\prime}$ with the added edges.

We will study two cases of adjacent channel use constraints. The first prevents the use of adjacent channels in the same cell simultaneously but allows any other form of adjacent channel use. All nondiagonal elements of the corresponding $B$ matrix are 1 and the diagonal elements are 2 . The second adjacent channel use constraint prevents the simultaneous use of adjacent channels in cells separated by a distance $\leq 1$, i.e., $b_{i j}=1$ iff $d(i, j)>1$ and $b_{i j}=2$ otherwise.

We have also studied two different traffic patterns: Uniform traffic (UT) pattern and the Nonuniform traffic (NUT) pattern. 
TABLE IV

A COMPARISON OF THE COMPLEXITIES OF DEORA's METHOD [2] AND OUR Method in the Case Where Adjacent Channel Use in the SAME CelL AND IN ADJACENT CELLS IS PROHIBITED

\begin{tabular}{|c|c|c|c|c|c|}
\hline \multirow{3}{*}{$\begin{array}{l}\text { No. of } \\
\text { cells }\end{array}$} & \multirow{3}{*}{$\begin{array}{l}\text { Cochannel } \\
\text { Interference } \\
\text { Threshold }\end{array}$} & \multicolumn{2}{|c|}{ Deora's method[2] } & \multicolumn{2}{|c|}{ Our method } \\
\hline & & \multicolumn{2}{|c|}{ Number of } & \multicolumn{2}{|c|}{ Number of } \\
\hline & & vars. & constrs. & vars & constrs \\
\hline 3 & $\left(\begin{array}{ll}0 & 1\end{array}\right)$ & 11 & 8 & 3 & 4 \\
\hline 7 & 0.15 & 63 & 24 & 11 & 8 \\
\hline 19 & 0.1 & 7368 & 311 & 86 & 20 \\
\hline 19 & 0.2 & 19097 & 832 & 249 & 20 \\
\hline 19 & 0.3 & 24131 & 1237 & 242 & 20 \\
\hline 19 & 0.375 & 25125 & 1322 & 188 & 20 \\
\hline 19 & 0.4 & 25244 & 1396 & 194 & 20 \\
\hline 19 & 0.5 & 25429 & 1431 & 167 & 20 \\
\hline 19 & 0.6 & 25465 & 1443 & 146 & 20 \\
\hline 19 & $2 / 3$ & 25467 & 1444 & 140 & 20 \\
\hline
\end{tabular}

TABLE V

THE LOWER Bounds ON CAPACITIES ObTAined Using OUR METHOD In the Case Where Adjacent Channel Use in the Same Cell AND IN ADJACENT CELLS IS PROHIBITED

\begin{tabular}{|r|l|l|l|l|l|l|l|}
\hline \multirow{2}{*}{$\begin{array}{c}\text { No. of } \\
\text { cells }\end{array}$} & $\begin{array}{c}\text { Cochannel } \\
\text { Interference } \\
\text { Threshold }\end{array}$ & \multicolumn{2}{|c|}{$r_{0}^{A l} / r_{0}^{A}$} & \multicolumn{2}{|c|}{$r_{0}^{A L}$} & \multicolumn{2}{|c|}{$r_{0}^{A}$} \\
\hline 3 & $(01)$ & 1.0 & & 0.75 & & 0.75 & \\
7 & 0.15 & 0.75 & & 0.875 & & 1.17 & \\
19 & 0.1 & 0.65 & 0.80 & 1.55 & 1.28 & 2.37 & 1.6 \\
19 & 0.2 & 0.76 & 0.80 & 2.11 & 1.6 & 2.78 & 2.0 \\
19 & 0.3 & 0.73 & 0.96 & 2.33 & 1.92 & 3.17 & 2.0 \\
19 & 0.375 & 0.80 & 1.0 & 2.52 & 2.0 & 3.17 & 2.0 \\
19 & 0.4 & 0.81 & 1.0 & 2.55 & 2.0 & 3.17 & 2.0 \\
19 & 0.5 & 0.90 & 1.0 & 2.85 & 2.0 & 3.17 & 2.0 \\
19 & 0.6 & 0.95 & 1.0 & 3.0 & 2.0 & 3.17 & 2.0 \\
19 & $2 / 3$ & 1.0 & 1.0 & 3.17 & 2.0 & 3.17 & 2.0 \\
\hline
\end{tabular}

In the former the same amount of traffic is offered in each cell, i.e., $p_{i}=1 / N$, for all $i$. In the latter the maximum traffic is offered in the central cell, one half of that in the next ring of cells, one third of that in the next ring, and so on. Nonuniform traffic pattern has been studied for the system with 19 cells only. For this system

$$
p_{i}= \begin{cases}1 / 24, & i \in\{1, \ldots, 4,7,8,12,13,16, \ldots, 19\}, \\ 1 / 16, & i \in\{5,6,9,11,14,15\}, \text { and } \\ 1 / 8, & i=10 .\end{cases}
$$

This model of nonuniform traffic pattern may be representative of cities where more traffic is offered in the center and less in the outskirts.

The results for the first case have been tabulated in Tables II and III and for the second case in Tables IV and V. The number of vertices in $G$ and $G^{\prime}$ have not been listed for the second case because the graph $G$ is completely disconnected in all these cases and we get $p_{\min }$ and, hence, $r_{0}^{A L}$ from the special case 2). The number of vertices in $G$ and $G^{\prime}$ are anyway the same as the corresponding ones in Table II.

In Table II, we have not listed the number of vertices of $G$ and $G^{\prime}$ for the 19-cell system with nonuniform traffic pattern for interference threshold $\geq 0.4$ because an optimal solution of LP2 in each of these cases is same as that for an interference threshold of 0.375. Thus, the graphs $G$ and $G^{\prime}$ are also the same as that for an interference threshold of 0.375 . We have listed the number of variables, constraints, and so on, in the linear program used in [2] in one of the cases for the 37 cell system. The numbers are even larger for the other cases for the 37 cell system when the interference threshold is higher. ${ }^{11}$

The following observations may be made from the tabulated data.

1) We know that $r_{0}^{A L} \leq r_{0}^{A} \leq r_{0}$. Thus, $r_{0}^{A L}$ is a good approximation to $r_{0}^{A}$ if $r_{0}^{A L} / r_{0}=\left(1+p / \sum_{j=1}^{M} m_{j}\right)^{-1}$ is close to 1 and is the same as $r_{0}^{A}$ if $r_{0}^{A L} / r_{0}=1$. Thus, Table III indicates that $r_{0}^{A L}$ is a very good approximation to $r_{0}^{A}$ and often gives the exact $r_{0}^{A}$ in the first case, i.e., when adjacent channels cannot be used simultaneously in the same cell only. Also the proximity of $r_{0}^{A L} / r_{0}$ to 1 indicates that $r_{0}^{A} \approx r_{0}$ in this case. Table II indicates that the computation of the exact value of $r_{0}^{A}$ as per [2] may be impossible in this case even for the system with 19 cells. We could compute the approximations for the system with 19 cells using no more than $0.4 \mathrm{~s}$ of system time on an IBM SP2 machine. Our computations took less than $0.5 \mathrm{~min}$ of system time on an IBM SP2 for the system with 37 cells.

2) Consider Table V, where we list the results for the case where adjacent channels cannot be used simultaneously in the same cell and in adjacent cells. The graph $G$ is completely disconnected in this case and $p_{\min }=\gamma_{G^{\prime}}$. Thus, $r_{0}^{A L} / r_{0}=(1+$ $\left.p / \sum_{j=1}^{M} m_{j}\right)^{-1}=0.5$. Thus, the value of $r_{0}^{A L} / r_{0}$ does not guarantee that our approximation is good. However, in this case the number of variables and constraints in the linear program used in [2] are not that large and we could compute $r_{0}^{A}$ as per [2] for systems with 3, 7, 19 cells. Comparison of $r_{0}^{A L}$ with $r_{0}^{A}$ indicates that $r_{0}^{A L}$ is reasonably close to $r_{0}^{A}$ in most of the cases but there is a significant difference in some of the cases. Better heuristics than those we have proposed here may be needed in such cases. The results indicate that $r_{0}$ (in this case $r_{0}=$ $2 r_{0}^{A L}$ ) is significantly higher than $r_{0}^{A}$. Our approximation is much easier to compute in this case even for the system with 19 cells. For the system with 37 cells again the computation of the exact value of $r_{0}^{A}$ as per [2] may be impossible.

\section{ApPRoximation to $T_{A}(r)$}

We now show how to approximate $T_{A}(r)$. The approach is similar to the that of the previous section. We start with the optimal solution of LP1 instead of LP2 here. The details follow.

Let LP1 have a rational optimal solution $\left(X_{1}^{O}, \ldots, X_{M}^{O}\right)$. Now let $G$ and $G^{\prime}$ be the graphs induced by the $M$-tuple $\left(X_{1}^{O}, X_{2}^{O}, \ldots, X_{M}^{O}\right)$. Clearly $X_{k}^{O}=m_{k} / \sum_{j=1}^{M} m_{j}$, for $1 \leq k \leq M$.

Theorem 3: Let $G^{\prime}$ become Hamiltonian upon the addition of $p$ edges. Then $T_{A}(r) \geq T(r) /\left(1+p / \sum_{j=1}^{M} m_{j}\right)$.

Proof: This proof is similar to that of Theorem 2. Let there be $n$ channels. Then $n=q\left(p+\sum_{j=1}^{M} m_{j}\right)+t$ for some $t$ and $q$ where $0 \leq t<\left(p+\sum_{j=1}^{M} m_{j}\right)$ and $q$ is a nonnegative integer (Euclidean division theorem). By Theorem 1 we have a fixed channel allocation algorithm allocating $q \sum_{j=1}^{M} m_{j} a_{i j}$

\footnotetext{
${ }^{11}$ From the Hamilton cycle, $r_{0}^{A L}=\left(1+p / \sum_{j=1}^{M} m_{j}\right)^{-1} r_{0}=4.5$. Using the fact that $r_{0}^{A}$ increases with increase in allowable interference threshold and $r_{0}^{A}=5.04$ for a lower value of the interference threshold, namely 0.375 , we get a better lower bound. We have similarly improved the lower bound for the 37 -cell system with an interference threshold of 0.5 .
} 
channels to the $i$ th cell for $1 \leq i \leq N$. Using the ATP and the independence of offered traffic it can be shown that this fixed channel allocation algorithm carries a traffic intensity of $\min \left(p_{i} r, \sum_{j=1}^{M} m_{j} a_{i j} /\left(p+\sum_{j=1}^{M} m_{j}\right)\right)$ in the asymptotic limit $(n \rightarrow \infty)$ in cell $i$ (refer to the Appendix for the proof). Let the total traffic intensity carried by this algorithm in the system be $T_{F C A}(r)$. Then

$$
\begin{aligned}
T_{F C A}(r) & =\sum_{i=1}^{N} \min \left(p_{i} r, \frac{\sum_{j=1}^{M} m_{j} a_{i j}}{p+\sum_{j=1}^{M} m_{j}}\right) \\
& \geq \sum_{i=1}^{N} \frac{\sum_{j=1}^{M} m_{j}}{p+\sum_{j=1}^{M} m_{j}} \min \left(p_{i} r, \frac{\sum_{j=1}^{M} m_{j} a_{i j}}{\sum_{j=1}^{M} m_{j}}\right) \\
& =\frac{\sum_{j=1}^{M} m_{j}}{p+\sum_{j=1}^{M} m_{j}} \sum_{i=1}^{N} \min \left(p_{i} r, \sum_{j=1}^{M} X_{j}^{O} a_{i j}\right) \\
& =\frac{\operatorname{since} X_{j}^{O}=m_{j} / \sum_{k=1}^{M} m_{k}}{p+\sum_{j=1}^{M} m_{j}} T(r) .
\end{aligned}
$$

Thus, $T_{A}(r) \geq T_{F C A}(r) \geq\left(\sum_{j=1}^{M} m_{j} /\left(p+\sum_{j=1}^{M} m_{j}\right)\right) T(r)$ $=T_{A L}(r)$, where the last equality defines $T_{A L}(r)$

Again, the lower bound is the tightest if $p=p_{\min }$. The techniques for finding $p_{\min }$ in the special cases and the approximation to $p_{\min }$ in the general case discussed in Section IV apply here. Certain other observations simplify the computation of $T_{A}(r)$, e.g., $T_{A}(r)=r$ for $r \leq r_{0}^{A L}$. (This follows from the definition of $r_{0}^{A}$ and the fact that $r_{0}^{A L} \leq r_{0}^{A}$.) Since $T_{A L}(r)=T(r) /\left(1+p / \sum_{j=1}^{M} m_{j}\right) \leq T_{A}(r) \leq T(r)$, one can be assured that the approximation is doing well if $p$ is small compared to $\sum_{j=1}^{M} m_{j}$. In general, it is much easier to compute $T_{A L}(r)$ than $T_{A}(r)$ as per [2].

We would also like to point out that since $T_{A}(r)=r$, for $r \leq r_{0}^{A L} \leq r_{0}^{A}$, if $r_{0}^{A L}$ is known then we can set $T_{A L}(r)=r$ for $r \leq r_{0}^{\bar{A} L}$.

Example V.1: Consider the system with seven cells described in Example III.1 (Fig. 2). The same model for interference is assumed as in Section III The cochannel reuse constraint and, hence, the maximal independent sets of the hypergraph modeling the cochannel reuse constraint remains the same as in Example III.1. LP1 gives the following solution:

$$
T(r)= \begin{cases}r, & \text { if } r \leq 7 / 4 \\ 1+3 r / 7, & \text { if } 7 / 4<r \leq 7 / 3 \\ 2, & \text { if } r \geq 7 / 3\end{cases}
$$

For

$$
r \leq 7 / 4, \quad X_{i}(r)= \begin{cases}r / 14, & \text { if } i \in\{a, b, \ldots, f\} \\ r / 7, & \text { if } i=g \\ 0, & \text { otherwise. }\end{cases}
$$

For

$$
\begin{aligned}
7 / 4<r \leq 7 / 3 & \\
X_{i}(r) & = \begin{cases}r / 14, & \text { if } i \in\{a, b, \ldots, f\} \\
1-3 r / 7, & \text { if } i=g, \\
0, & \text { otherwise. }\end{cases}
\end{aligned}
$$

For

$$
r \geq 7 / 3, \quad X_{i}(r)= \begin{cases}1 / 6, & \text { if } i \in\{a, b, \ldots, f\} \\ 0, & \text { otherwise. }\end{cases}
$$

Throughout, in this example, we shall assume that $r \in Q$. We shall consider two different adjacent channel use constraints as in Example III.1.

Case 1) Adjacent channels cannot be used simultaneously only in the same cell. We have found in Example IV.2 that $r_{0}^{A}=r_{0}^{A L}=7 / 4$. So $T_{A L}(r)=r$, for $r \leq 7 / 4$. Let $h=\operatorname{LCM}(r, 14-6 r)$. For $7 / 4<r<7 / 3$

$$
m_{i}= \begin{cases}h \frac{r}{\min (r, 14-6 r)}, & \text { if } i \in\{a, b, \ldots, f\} \\ h \frac{14-6 r}{\min (r, 14-6 r)}, & \text { if } i=g \\ 0, & \text { otherwise. }\end{cases}
$$

where $h=\operatorname{LCM}(r, 14-6 r)$.

Since $m_{i} \neq 0, i \in\{a, b, \ldots, g\}, G$ is given by Fig. 5(a). $G^{\prime}$ is always Hamiltonian in this range. Let $m_{i}=x, i \in\{a, b, \ldots, f\}$ and $m_{g}=y$. $V_{G}^{\prime}=\left\{a_{1}, a_{2}, \ldots, a_{x}, b_{1}, b_{2}, \ldots, b_{x}, \ldots f_{1}, f_{2}\right.$, $\left.\ldots, f_{x}, g_{1}, g_{2}\right\}, \ldots, g_{y} . a_{1} b_{1} \cdots f_{1} a_{2} b_{2} \cdots f_{2} \ldots$ $a_{x} b_{x} \cdots f_{x} a_{1}$ is a cycle in $G^{\prime}$. Now insert $g_{1}$ between $a_{1}$ and $b_{1}, g_{2}$ between $b_{1}$ and $c_{1}$, and so on in the above cycle till the $g_{i}$ s are exhausted. The $g_{i}$ s will be exhausted before one reaches $b_{\lceil x / 3\rceil}$ since $0<y<$ $2 x$ for $7 / 4<r<7 / 3$. This gives a Hamilton cycle in $G^{\prime}$ since $g_{i}$ and $p_{j}, p \in\{a, b, \ldots, f\} 1 \leq i \leq y$ and $1 \leq j \leq x$ are connected by an edge in $G^{\prime}$. Thus, $p_{\min }=0 . T_{A L}(r)=T(r)$ for $7 / 4<r<7 / 3$. For $r \geq 7 / 3, m_{i}=1, i \in\{a, b, \ldots, f\}$ and $m_{i}=0$ otherwise. $G$ and $G^{\prime}$ are given by Fig. 5(c). $G^{\prime}$ is clearly Hamiltonian. The Hamilton cycle is abcdefa. Thus, $T_{A L}(r)=T(r), r>7 / 4$. Thus, $T_{A L}(r)=T(r)$ for all $r$. Hence, $T_{A}(r)=T(r)$ for all $r$. Refer to Fig. 7 for $T_{A L}(r), T_{A}(r)$ and $T(r)$. Recall that we had observed earlier that $r_{0}^{A L}$ is very close to $r_{0}$ and, hence, to $r_{0}^{A}$ for the same adjacent channel use constraint as in this case and we find that $T_{A L}(r)=T_{A}(r)=T(r)$ for the system with seven cells. Hence, we expect that $T_{A L}(r)$ will track $T(r)$ and hence $T_{A}(r)$ very closely even for larger systems with the same adjacent channel use constraint as in this example. 


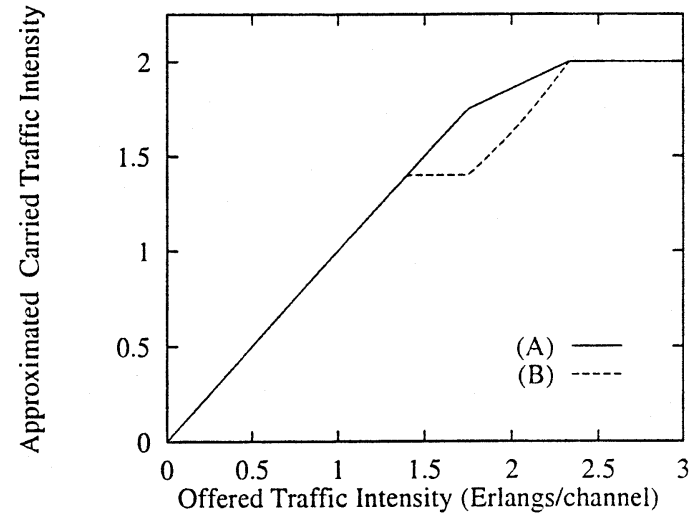

Fig. 7. The curve (A) gives $T_{A L}(r), T(r)$ and $T_{A}(r)$ for Example II.3.1. The curve (A) also gives $T(r)$ for Example II.3.2, while the curve (B) gives $T_{A L}(r)$ for Example II.3.2. $T_{A}(r)$ is between curves (A) and (B) in this case.

Case 2) Again assume that adjacent channels cannot be used simultaneously in the same cell and also that cell 7 cannot use a channel if its adjacent channel is being used in either cell 2, 4, or 6. We know from Example IV.2 that $r_{0}^{A L}=7 / 5$. Thus, $T_{A L}(r)=r$ for $r \leq$ $7 / 5$. For $7 / 5<r \leq 7 / 4$,

$$
m_{i}= \begin{cases}1, & \text { if } i \in\{a, b, \ldots, f\} \\ 2, & \text { if } i=g \\ 0, & \text { otherwise. }\end{cases}
$$

Refer to Fig. 6(a) and (b) for $G$ and $G^{\prime}$ respectively. $G^{\prime}$ is not Hamiltonian. However, it becomes Hamiltonian when edges are added between the pairs of vertices $(g 1, b)$ and $(d, g 2)$. Thus, $p_{\min } \leq 2$ and $T_{A L}(r)=0.8 T(r)$, for $7 / 5<r \leq 7 / 4$. $b c d g 2 e f a g 1 b$ is a Hamilton cycle in $G^{\prime}$ with the added edges. However, we can get a better upper bound by observing that $T_{A L}(7 / 5)=7 / 5$ and $T_{A L}(r) \geq T_{A L}\left(r^{\prime}\right)$, if $r \geq r^{\prime}$. Using this $T_{A L}(r)=7 / 5$, for $7 / 5<r \leq 7 / 4$. For $7 / 4<r<7 / 3, m_{i}$ s are given by those in case 1 for the same range of $r$. $G$ is given by Fig. 6(a). $V_{G}^{\prime}$ is also the same as that given in case 1 for the same range of $r . G^{\prime}$ is not, in general, Hamiltonian. However $G^{\prime}$ can be made Hamiltonian by adding edges between the pairs of vertices $\left(g_{1}, b_{1}\right),\left(g_{2}, d_{1}\right),\left(g_{3}, b_{2}\right)$, $\left(g_{4}, d_{2}\right)$, and so on until the $g_{i}$ s are exhausted. (All $g_{i}$ s will be exhausted because $0<y<2 x$ here, where $x, y$ are as defined in case 1 for the same range of $r$.) $b_{1} c_{1} d_{1} g_{2} e_{1} f_{1} a_{1} g_{1} b_{2} c_{2} d_{2} g_{4} e_{2} f_{2} a_{2} g_{3}$ $\cdots b_{l} c_{l} d_{l} g_{2 l} e_{l} f_{l} a_{l} g_{2 l-1} \cdots b_{x} c_{x} d_{x} e_{x} f_{x} a_{x} b_{1}$ is a Hamilton cycle in $G^{\prime}$ with the added edges. Thus, for $7 / 4<r<7 / 3, p_{\min } \leq y$ and

$$
\begin{aligned}
T_{A L}(r) & =\frac{T(r)}{1+\frac{y}{\sum_{i \in\{a, \ldots, g\}}^{y} m_{i}}} \\
& \left.=\frac{T(r)}{2-3 r / 7} \quad \text { (using the values of } y \text { and } m_{i} \mathrm{~s}\right) \\
& \left.=\frac{7+3 r}{14-3 r} \quad \text { [using the value of } T(r)\right] .
\end{aligned}
$$

For $r \geq 7 / 3, m_{i}$ s are given by those in case 1 for the same range of $r . G$ and $G^{\prime}$ are given by Fig. 5(c). $G^{\prime}$ is clearly Hamiltonian. abcdef $a$ is the Hamilton cycle. Thus, $T_{A L}(r)=T(r)$. Summarizing

$$
T_{A L}(r)= \begin{cases}r, & \text { if } r \leq 7 / 5 \\ 7 / 5, & \text { if } 7 / 5<r \leq 7 / 4 \\ \frac{7+3 r}{14-3 r}, & \text { if } 7 / 4<r<7 / 3 \\ 2, & \text { if } r \geq 7 / 3 .\end{cases}
$$

\section{Approximation in the Presence of Arbitrary RESTRICTIONS ON SIMULTANEOUS USE OF ANY Two DIFFERENT CHANNELS}

Simultaneous use of any two channels can produce interference even if they are not the same or adjacent. In most cases, the filter responses are good enough so that the interference produced by nonadjacent channels is negligible. Nevertheless, sometimes there may be restrictions on the simultaneous use of nonadjacent channels also. As mentioned before [2] formulates linear programs for the computation of $T_{A}(r)$ and $r_{0}^{A}$ in the presence of any such restriction but these linear programs are intractable because of the large number of variables and constraints even for systems of moderate size, e.g., a system with 19 cells. ${ }^{12}$ We can extend our approximations to this general case under certain special circumstances.

The cochannel reuse constraints are modeled by a hypergraph as before. The channels are numbered $1,2, \ldots, n$. The distance between channels numbered $i, j$ is defined to be $|i-j|$. The numbering should be such that this distance is proportional to the actual separation between them in the radio spectrum. The adjacent channel use constraints are modeled by a matrix $B$, which is defined slightly differently from the corresponding definition in Section I. $B$ is a $N \times N$ matrix, such that if cells $i, j$ can use adjacent channels simultaneously, then $b_{i j}=1$. Otherwise, $b_{i j}$ is the minimum distance between the channels that can be used simultaneously in cells $i$ and $j$. Note that if the restriction is only on adjacent channel use, as assumed in Sections IV and III, then the elements of $B$ are 1 and 2. (This agrees with the definition of $B$ in Section IV.)

A graph $D$ is induced by an $M$-tuple $\left(X_{1}, X_{2}, \ldots, X_{M}\right)$ as follows. Let $\left(X_{1}, X_{2}, \ldots, X_{M}\right)$ be any $M$-tuple, such that $X_{j}$ is a nonnegative rational number and $M$ is the number of maximal independent sets of the hypergraph modeling the cochannel reuse constraints of the cellular system. Let $X_{k_{1}}, X_{k_{2}}, \ldots, X_{k_{V}}$ be the ones with nonzero values. Let $X_{k_{1}}: X_{k_{2}}: \cdots: X_{k_{V}}=m_{k_{1}}: m_{k_{2}}: \cdots: m_{k_{V}}$, where $m_{k_{1}}, m_{k_{2}}, \ldots, m_{k_{V}}$ are relatively prime positive integers. If $X_{j}=0, m_{j}=0$. Form a weighted graph $D=\left(V_{D}, E_{D}\right)$ as follows: $V_{D}=\left\{v_{k_{1} 1}, \ldots, v_{k_{1} m_{k_{1}}}, v_{k_{2}}, \ldots, v_{k_{2} m_{k_{2}}}, \ldots\right.$, $\left.v_{k_{V} 1}, \ldots, v_{k_{V} m_{k_{V}}}\right\}$.

\footnotetext{
${ }^{12}$ If there are restrictions on the simultaneous use of $c$ channels, $\Omega \subseteq \tau \times$ $\tau \times \cdots \times \tau, c$ times. Refer to the relevant discussion in Section I of the work in [2]. $|\tau|$ is large and $|\Omega|$ is very large making the number of variables extremely large.
} 


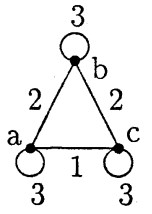

Fig. 8. The weighted $D$ graph for a system with three cells. The numbers against the edges indicate their weights.

There is an edge between any two vertices $v_{i}$ and $v_{j}$ (even if $v_{i}=v_{j}$ ). The weight of an edge between the vertices $v_{k_{l} a}$ and $v_{k_{t} b}$ is

$$
\begin{aligned}
w\left(v_{k_{l} a}, v_{k_{t} b}\right) & =\max _{1 \leq i, j \leq N} b_{i j} a_{i k_{l}} a_{j k_{t}} \\
& =\max _{\substack{1 \leq i \leq N, a_{i k_{l}}=1 \\
1 \leq j \leq N, a_{j k_{t}}=1}} b_{i j} .
\end{aligned}
$$

Example VI.1: Consider the system with three cells shown in Fig. 1. Let the minimum separation between channels used simultaneously in the same cell be 3 , i.e., cell 1 cannot use channels 1,2 or 1, 3 simultaneously and so on. Adjacent cells cannot use adjacent channels simultaneously. Any other cells can use adjacent channels simultaneously. Let there be no cochannel reuse in the system, i.e., there are 3 maximal independent sets $a, b, c$ each consisting of a single cell. $a=\{1\}, b=\{2\}, c=\{3\}$

$$
B=\left[\begin{array}{lll}
3 & 2 & 1 \\
2 & 3 & 2 \\
1 & 2 & 3
\end{array}\right] .
$$

The graph $D$ induced by $(2,2,2)$ is shown in Fig. 8 .

We present the results as follows.

Theorem 4: Let $D$ be the weighted graph induced by some $M$-tuple $\left(X_{1}, X_{2}, \ldots, X_{M}\right)$. Let $D$ satisfy the triangle inequality. ${ }^{13}$ Let there be a Hamilton cycle in $D$ of weight ${ }^{14}$ $L$. Let $w_{\max }$ be the weight of an edge which has the maximum weight amongst all edges in the Hamilton cycle. Let $\left(q L-w_{\max }+1\right)^{+}$channels be available. Then there exists a fixed channel allocation algorithm that allocates $q \sum_{j=1}^{M} m_{j} a_{i j}$ channels to the $i$ th cell, for $1 \leq i \leq N$, for any nonnegative integer $q$.

Proof: This proof is along the same lines as that of Theorem 1. Again if $q=0$ the lemma is trivially true $\left(w_{\max } \geq 1\right)$. Let $q>0$. Let the Hamilton cycle of weight $L$ be $v_{i_{0} j_{0}}, v_{i_{1}, j_{1}}, \ldots, v_{i_{U-1}, j_{U-1}}, v_{i_{0} j_{0}} . U=\sum_{j=1}^{M} m_{j}$. Without loss of generality $w\left(v_{i_{U-1}}, j_{U-1}, v_{i_{0} j_{0}}\right)=w_{\max }$. If the algorithm given in the proof of Theorem 1 is slightly modified, it allocates $q m_{j}$ channels to the $j$ th maximal independent set satisfying the cochannel reuse and the adjacent channel use constraints, using $\left(q L-w_{\max }+1\right)^{+}$channels. The following modification is required in step 4$)$ :

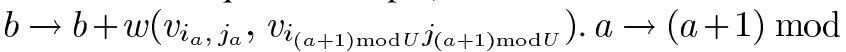
$U$. Go to step 2).

Clearly, this allocation satisfies the cochannel reuse constraints. Let cell $i$ get channel $k_{1}$ and cell $j$ get channel $k_{2}$. Let channel $k_{1}$ be allocated to the $s$ th maximal independent set and channel $k_{2}$ be allocated to the th maximal independent set. Thus, cell $i$ is in the $s$ th maximal independent set and cell $j$ is in the $t$ th maximal independent set. Note that $\left|k_{1}-k_{2}\right|$ is equal to the total weight of at least one of the paths between $v_{k_{1}}$ and

\footnotetext{
${ }^{13} w\left(v_{1}, v_{2}\right) \leq w\left(v_{1}, v_{3}\right)+w\left(v_{3}, v_{2}\right)$

${ }^{14}$ The weight of a Hamilton cycle in a weighted graph is the sum of the weights of the edges in the cycle.
}

$v_{k_{2}}$ on the Hamilton cycle. Because of the triangle inequality, the weight of both the paths on the Hamilton cycle is greater than or equal to $w\left(v_{k_{1}}, v_{k_{2}}\right)$ and this weight is greater than or equal to $b_{i j}$, the minimum separation between the channels which can be simultaneously used in these cells. Thus, this allocation satisfies the adjacent channel use constraints. This is the advertised fixed channel allocation.

The following Corollary follows from Theorem 4.

Corollary 2: Let a fixed channel allocation that allocates $n_{i}$ channels to the $i$ th cell be desired. Let $D$ be the graph induced by an optimal solution of the ILP $\left(X_{1}^{O}, X_{2}^{O}, \ldots, X_{M}^{O}\right)$. Let $q=$ g.c.d. of nonzero $X_{j}^{O}$ s if there exists at least one nonzero $W_{j}^{O}$ and let $q=0$ otherwise. Let there be a Hamilton cycle in $D$ of weight $L . w_{\max }$ is the weight of an edge which has the maximum weight amongst all edges in the Hamilton cycle. If $D$ satisfies the triangle inequality, $\left(q L-w_{\max }+1\right)^{+}$channels are sufficient to achieve the required fixed channel allocation.

Theorem 5: Let $D$ be the graph induced by a rational optimal solution of LP2, $\left(X_{1}^{O}, X_{2}^{O}, \ldots, X_{M}^{O}\right)$. Let there be a Hamilton cycle in $D$ of weight $L$. If $D$ satisfies the triangle inequality, $r_{0}^{A} \geq r_{0} \sum_{j=1}^{M} m_{j} / L$

Proof: The proof follows from Theorem 4, Lemmas 1 and 2 along the same lines as the proof of Theorem 1 follows from Theorem 1, and Lemmas 1 and 2.

Remarks: $r_{0} \sum_{j=1}^{M} m_{j} / L \leq r_{0}^{A} \leq r_{0}$. The lower bound is tightest if $L=L_{\min }$, where $L_{\min }$ is the weight of the minimum weight Hamilton cycle in the graph $D$ induced by $\left(X_{1}^{O}, X_{2}^{O}, \ldots, X_{M}^{O}\right)$, a rational optimal solution of LP2.

Theorem 6: Let $D$ be the graph induced by a rational optimal solution of LP1, $\left(X_{1}^{O}, X_{2}^{O}, \ldots, X_{M}^{O}\right)$. Let there be a Hamilton cycle in $D$ of weight $L$. If $D$ satisfies the triangle inequality, $T_{A}(r) \geq T(r) \sum_{j=1}^{M} m_{j} / L$.

The proof follows the same lines as that of Theorem 3 .

Remarks: $T(r) \sum_{j=1}^{M} m_{j} / L \leq T_{A}(r) \leq T(r)$. Again the lower bound is the tightest if $L=L_{\min }$, where $L_{\min }$ is the weight of the minimum weight Hamilton cycle in the graph $D$ induced by $\left(X_{1}^{O}, X_{2}^{O}, \ldots, X_{M}^{O}\right)$, a rational optimal solution of LP1. Note that if the restriction is only on adjacent channel use, as assumed in Sections IV and III, then the elements of $B$ are 1 and 2. (This agrees with the definition of $B$ in Section IV.) This makes $D$ always satisfy the triangle inequality in this case. Also the lower bounds with $L_{\min }$ given by Theorems 5 and 6 turn out to be the same as those given by Theorems 2 and 3, respectively, with $p_{\min }$ in these cases. The same observation applies for the results of Corollaries 1 and 2.

The problem of finding the minimum weight Hamilton cycle in $D$ is the same as the traveling salesman problem which is NP-complete. However if $D$ satisfies the triangle inequality, there are some polynomial time algorithms which will produce, within known bounds, an approximation to the traveling salesman problem [9]. Thus, this technique is useful, whenever $D$ satisfies the triangle inequality, to get an estimate of $r_{A}^{0}$ and $T_{A}(r)$, more so because the exact values of these quantities are intractable even for systems of reasonably small size. The generalization for the case in which $D$ does not satisfy triangle inequality is complicated. This is a topic for future research.

We would like to mention that the results in this section present our preliminary research in this area. Nevertheless, these results 
indicate promising directions for extending the approximations toward the general case of arbitrary restrictions for simultaneous use of any two channels. We hope that this research will stimulate further interest in the arbitrary generalizations.

\section{CONCLUSION AND SUMmARY}

We now summarize the contributions of this paper. There are various performance bounds for a cellular system which are useful from the theoretical as well as from the network operators' point of view. The computation of the exact values of these performance bounds in the presence of cochannel reuse and adjacent channel use constraints is difficult or rather impossible even for systems of reasonably small size. We have developed approximations to these performance bounds in the presence of cochannel reuse and adjacent channel use constraints which are computationally much simpler. These approximations track very closely the actual performance bounds in most cases. We have also presented good heuristics for the problem of finding the minimum number of channels necessary for achieving any given fixed channel allocation algorithm in the presence of cochannel reuse and adjacent channel use constraints.

We make one observation before conclusion: we have made all computations in two steps. The LPs or the ILP take care of the cochannel reuse constraints and the graph theoretic approach takes care of the adjacent channel use constraint. The advantage of this modularization is that if for some reason the adjacent channel use constraints change but the cochannel reuse constraints remain the same, then only the graph theoretic computations need be repeated. This is helpful because for actual systems both the ILP and the LPs may be computationally quite intensive.

\section{APPENDIX}

Lemma 1: Let the carried traffic in a one cell system be denoted by $C\left(r_{n}, h_{n}\right)$ when $h_{n}$ channels are allocated to the cell and $r_{n}$ is the offered traffic in the cell. $\lim _{n \rightarrow \infty} C\left(r_{n}, h_{n}\right) / n=$ $\min (r, h)$ if $\lim _{n \rightarrow \infty} r_{n} / n=r, \lim _{n \rightarrow \infty} h_{n} / n=h$ and $h>0$.

Proof: This proof follows easily from the ATP introduced in [4]. We give the proof here for the sake of completeness

$$
\begin{aligned}
\lim _{n \rightarrow \infty} \frac{C\left(r_{n}, h_{n}\right)}{n}= & \lim _{n \rightarrow \infty} \frac{h_{n}}{n} \lim _{n \rightarrow \infty} \frac{C\left(r_{n}, h_{n}\right)}{h_{n}} \\
= & h \lim _{h_{n} \rightarrow \infty} \frac{C\left(r_{n}, h_{n}\right)}{h_{n}} \\
& \cdot\left(h_{n} \rightarrow \infty \text { as } n \rightarrow \infty \text { since } h>0\right) . \\
= & h \min \left(\lim _{h_{n} \rightarrow \infty} \frac{r_{n}}{h_{n}}, 1\right) \quad \text { by the ATP } \\
= & h \min \left(\frac{r}{h}, 1\right) \\
= & \min (r, h) .
\end{aligned}
$$

Lemma 2: Let $n=q\left(p+\sum_{j=1}^{M} m_{j}\right)+t$ channels be available where $0 \leq t<\left(p+\sum_{j=1}^{n} m_{j}\right)$ and $q$ is a nonnegative integer. The fixed channel allocation algorithm which allocates $q \sum_{j=1}^{M} m_{j} a_{i j}$ channels to the $i$ th cell carries a traffic intensity of $\min \left(p_{i} r, \sum_{j=1}^{M} m_{j} a_{i j} /\left(p+\sum_{j=1}^{M} m_{j}\right)\right)$ in the asymptotic limit $(n \rightarrow \infty)$ in the $i$ th cell.

Proof: As argued in [4], because of the independence of the offered traffic and the fixed channel allocation algo- rithm, each cell behaves as a one cell system with offered load $p_{i} r n$ and $q \sum_{j=1}^{M} m_{j} a_{i j}$ channels. Thus, the carried traffic intensity in the $i$ th cell is $C\left(p_{i} r n, q \sum_{j=1}^{M} m_{j} a_{i j}\right) / n$. If $\sum_{j=1}^{M} m_{j} a_{i j}=0, C\left(p_{i} r n, q \sum_{j=1}^{M} m_{j} a_{i j}\right) / n=0$, for each $n$. Thus, $\lim _{n \rightarrow \infty} C\left(p_{i} r n, q \sum_{j=1}^{M} m_{j} a_{i j}\right) / n=$ $0=\min \left(p_{i} r, \sum_{j=1}^{M} m_{j} a_{i j} /\left(p+\sum_{j=1}^{M} m_{j}\right)\right.$. Let $\sum_{j=1}^{M} m_{j} a_{i j}>0$. Then $p_{i} r n / n \stackrel{n \rightarrow \infty}{\longrightarrow} p_{i} r$ and $\lim _{n \rightarrow \infty}(q / n)=$ $1 /\left(p+\sum_{j=1}^{M} m_{j}\right)$ since $0 \leq t<\left(p+\sum_{j=1}^{M} m_{j}\right)$ and $p, m_{j}, a_{i j}, M$ are fixed constants independent of $n$. Thus, $\lim _{n \rightarrow \infty}\left(q \sum_{j=1}^{M} m_{j} a_{i j} / n\right)=\sum_{j=1}^{M} m_{j} a_{i j} /(p+$ $\left.\sum_{j=1}^{M} m_{j}\right)>0$. Thus, $\lim _{n \rightarrow \infty} C\left(p_{i} r n, q \sum_{j=1}^{M} m_{j} a_{i j}\right) / n=$ $\min \left(p_{i} r, \sum_{j=1}^{M} m_{j} a_{i j} /\left(p+\sum_{j=1}^{M} m_{j}\right)\right)$ by Lemma 1 .

\section{REFERENCES}

[1] S. Sarkar and K. N. Sivarajan, "Hypergraph models for cellular mobile communication systems," IEEE Trans. Veh. Technol., to be published.

[2] S. Deora, "Channel assignment algorithms in cellular radio networks," Ph.D. dissertation, California Inst. Technol., Pasadena, May 1995.

[3] V. H. MacDonald, "The cellular concept," Bell Syst. Tech. J., vol. 58, no. 1, Jan. 1979.

[4] R. J. McEliece and K. N. Sivarajan, "Performance limits for channelized cellular telephone systems," IEEE Trans. Inform. Theory, vol. 40, pp. 21-34, Jan. 1994.

[5] S. Sarkar, "Hypergraph models, fairness and pricing in cellular mobile communication," Elect. Commun. Eng. Dept., Indian Inst. Sci., Bangalore, India, M.E. Project Rep., 1996.

[6] C. Berge, Hypergraphs. Amsterdam, The Netherlands: North-Holland, 1989.

[7] K. N. Sivarajan, "Performance limits for cellular systems with handoffs," in Proc. 31st Annu. Allerton Conf. Communications, Control, and Computing, vol. 19, 1993, pp. 887-897.

[8] J. Franklin, Methods of Mathematical Economics. New York: Springer-Verlag, 1980.

[9] A. Gibbons, Algorithmic Graph Theory. Cambridge, U.K.: Cambridge Univ. Press, 1985

[10] J. Bondy and U. Murthy, Graph Theory with Applications. New York: Macmillan, 1976.

[11] F. Harary, Graph Theory. Reading, MA: Addison-Wesley, 1994.

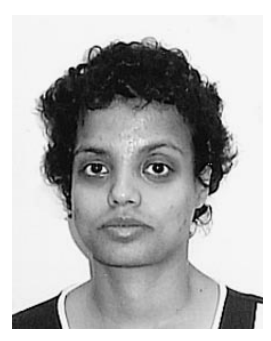

Saswati Sarkar received the Master of Engineering degree in electrical communication engineering from the Indian Institute of Science, Bangalore, India, in 1996 and the Ph.D. degree in electrical and computer engineering from the University of Maryland, College Park, in 2000.

She is currently an Assistant Professor with the Department of Electrical and Systems Engineering, University of Pennsylvania, Philadelphia. Her research interests are in resource allocation and performance analysis in communication networks.

Kumar N. Sivarajan (M'88) received the B.Tech. degree in electrical engineering (electronics) from the Indian Institute of Technology, Madras, in 1987 and the M.S. and Ph.D. degrees in electrical engineering from the California Institute of Technology, Pasadena, in 1988 and 1990, respectively.

From 1990 to 1994, he was with the IBM Thomas J. Watson Research Center, Yorktown Heights, NY. From 1994 to 2000, he was with the Electrical Communication Engineering Department, Indian Institute of Science, Bangalore. Since May 2000, he has been Chief Technology Officer of Tejas Networks, Bangalore. He is coauthor of Optical Networks: A Practical Perspective (New York: Morgan Kaufmann, 1998).

Dr. Sivarajan has served as an Editor of the IEEE/ACM TRANSACTIONS ON NETWORKING. He is an Associate of the Indian Academy of Sciences, the recipient of the Young Engineer Award from the Indian National Academy of Engineering, and the Swarnajayanti Fellowship from the Department of Science and Technology, Government of India. He was the recipient of the IEEE Charles LeGeyt Fortescue Fellowship for the academic year 1987-1988, the IEEE Communications Society 1996 William R. Bennett Prize Paper Award, and the 1997 IEEE W. R. G. Baker Prize Paper Award. 